\title{
On Convergence of the Inexact Rayleigh Quotient Iteration with the Lanczos Method Used for Solving Linear Systems*
}

\author{
Zhongxiao $\mathrm{Jia}^{\dagger}$
}

\begin{abstract}
For the Hermitian inexact Rayleigh quotient iteration (RQI), the author has established new local general convergence results, independent of iterative solvers for inner linear systems. The theory shows that the method locally converges quadratically under a new condition, called the uniform positiveness condition. In this paper we first consider the local convergence of the inexact RQI with the unpreconditioned Lanczos method for the linear systems. Some attractive properties are derived for the residuals, whose norms are $\xi_{k+1}$ 's, of the linear systems obtained by the Lanczos method. Based on them and the new general convergence results, we make a refined analysis and establish new local convergence results. It is proved that the inexact RQI with Lanczos converges quadratically provided that $\xi_{k+1} \leq \xi$ with a constant $\xi \geq 1$. The method is guaranteed to converge linearly provided that $\xi_{k+1}$ is bounded by a small multiple of the reciprocal of the residual norm $\left\|r_{k}\right\|$ of the current approximate eigenpair. The results are fundamentally different from the existing convergence results that always require $\xi_{k+1}<1$, and they have a strong impact on effective implementations of the method. We extend the new theory to the inexact RQI with a tuned preconditioned Lanczos for the linear systems. Based on the new theory, we can design practical criteria to control $\xi_{k+1}$ to achieve quadratic convergence and implement the method more effectively than ever before. Numerical experiments confirm our theory.
\end{abstract}

Keywords. Hermitian, inexact RQI, convergence, inner iteration, outer iteration, unpreconditioned Lanczos, tuned preconditioned Lanczos

AMS subject classifications. $65 \mathrm{~F} 15,65 \mathrm{~F} 10,15 \mathrm{~A} 18$

\section{Introduction}

We consider the problem of computing an eigenvalue $\lambda$ and the associated eigenvector $x$ of a large and possibly sparse Hermitian matrix $A \in \mathbb{C}^{n \times n}$, i.e.,

$$
A x_{i}=\lambda_{i} x_{i}, i=1,2, \ldots, n,
$$

where $\left(\lambda_{i}, x_{i}\right), i=1,2, \ldots, n$ are the eigenpairs with $\left\|x_{i}\right\|=1$ in the 2 -norm. Throughout the paper, we are interested in the eigenvalue $\lambda_{1}$ closest to a target $\sigma$ and its corresponding eigenvector $x_{1}$ in the sense that

$$
\left|\lambda_{1}-\sigma\right|<\left|\lambda_{2}-\sigma\right| \leq \cdots \leq\left|\lambda_{n}-\sigma\right|
$$

${ }^{*}$ Supported by National Basic Research Program of China 2011 CB302400 and the National Science Foundation of China (No. 11071140).

${ }^{\dagger}$ Department of Mathematical Sciences, Tsinghua University, Beijing 100084, People’s Republic of China, jiazx@tsinghua.edu.cn 
Suppose that $\sigma$ is between $\lambda_{1}$ and $\lambda_{2}$. Then (2) means

$$
\left|\lambda_{1}-\sigma\right|<\frac{1}{2}\left|\lambda_{1}-\lambda_{2}\right|
$$

There are a number of methods for solving this kind of problem, such as inverse iteration [13, Rayleigh quotient iteration (RQI) [13], the Lanczos method and its shift-invert variant [13, the Davidson method and the Jacobi-Davidson method [18, 20]. However, except the standard Lanczos method, these methods and shift-invert Lanczos involve the solution of a possibly ill-conditioned linear system at each iteration. This is generally very difficult and even impractical by a direct solver since a factorization of a shifted $A$ may be too expensive. So one generally resorts to iterative solvers for the linear systems, called inner iterations. We call updates of approximate eigenpairs outer iterations. A combination of them yields an inner-outer iterative eigensolver, also called an inexact eigensolver.

For $A$ Hermitian, general local convergence theory on the inexact RQI can be found in Smit [16, van den Eshof [19], Simoncini and Eldén [15], Berns-Müller and Spence [1] and Freitag and Spence [4, 7]. Berns-Müller and Spence [2] have extended the theory of [1] to the case that $A$ is non-Hermitian. For $A$ Hermitian and non-Hermitian, Xue and Elman [21] and Xue and Syzld [22] have further analyzed the local convergence of the inexact RQI and provided new insights into tuning a preconditioner, which is for efficient Krylov inner solves. The idea of tuning a preconditioner was initially proposed in [15] and then extended in [1, 2] and improved in [4, 7. Let $\left\|r_{k}\right\|$ be the residual norm of the approximate eigenpair at outer iteration $k$. The mentioned papers except [22] have proved that the inexact RQI converges cubically if $\xi_{k+1}=O\left(\left\|r_{k}\right\|\right)$ and quadratically if $\xi_{k+1} \leq \xi<1$ with a constant $\xi$ not near one. In [22], Xue and Syzld have given a new local convergence analysis, showing that the inexact RQI may demonstrate cubic and quadratic asymptotic convergence rates, respectively, for Hermitian and non-Hermitian problems, if the shifted linear systems are solved by a Krylov subspace method with a tuned preconditioner to a reasonably small fixed tolerance $\xi_{k+1} \leq \xi<1$. However, their claims hold only under a crucial assumption that the factor $\alpha_{m}$ in their main result (3.2) must be bounded by a moderate constant. However, it is not clear when this assumption is satisfied. They have given some qualitative but rough and non-rigorous arguments on $\alpha_{m}$, trying to show that $\alpha_{m}$ is bounded. In fact, the size of $\alpha_{m}$ is closely related to that of $\xi_{k+1}$, as was implicit from their arguments. Furthermore, their arguments implicitly indicate that $\alpha_{m}$ is guaranteed to be moderate only when $\xi_{k+1}$ is sufficiently small, and there is no evidence that a reasonably small fixed $\xi_{k+1}$ is enough. So (3.2) in Theorem 3.1 of [22] may not mean the cubic asymptotic convergence if the shifted linear systems are solved by a tuned preconditioned Krylov subspace method to a reasonably small fixed tolerance $\xi_{k+1} \leq \xi<1$. Particularly, if the Lanczos method is used for solving shifted inner linear systems, it is typical that $\alpha_{m}$ can be very large and $\xi_{k+1}$ may be bigger than one for some inner iterations when the linear systems are indefinite 1

Simoncini and Eldén [15] are the first to observe that poor approximate solutions of the linear systems may be much improved approximations to the desired eigenvector $x_{1}$. In our notation, their observation qualitatively means that $\xi_{k+1}$ can be near one when MINRES is used. More generally, a remarkable feature for the convergence of the inexact RQI with MINRES (or its mathematically equivalent version the conjugate residual method) is that $\xi_{k+1}$ is allowed not small. Xue and Elman [21] have given a qualitative mathematical justification on this phenomenon. We must point out that their results have also shown clearly that although the convergence of the inexact RQI with MINES allows $\xi_{k+1}$ near

\footnotetext{
${ }^{1}$ I have communicated with Dr Fei Xue, one author of [22. He has agreed that (i) their arguments on $\alpha_{m}$ are non-rigorous and more experimental and (ii) the condition of the cubic convergence result is not yet clear and how small $\xi_{k+1}$ should be is being under consideration.
} 
one, $\xi_{k+1}$ too near one is prohibited. Quantitatively, however, how near one $\xi_{k+1}$ is allowed is unknown. The observation by Simoncini and Eldén and the work followed may have led to some serious misunderstanding or wrong impressions that the size of $\xi_{k+1}$ plays no role in the convergence of the inexact RQI. In fact, this is completely wrong, and the size of $\xi_{k+1}$ does play a crucial role in the convergence and convergence rates of the inexact RQI. It can be easily observed that in literature all the convergence conditions involve $\xi_{k+1}$ either explicitly or implicitly, so do stopping criteria. They all assume $\xi_{k+1}<1$ first and then impose further restrictions to $\xi_{k+1}$ either explicitly or implicitly for achieving a desired convergence rate. This is easily understandable since an approximate solution corresponds to a unique $\xi_{k+1}$. Mathematically, any result on approximate solutions can be definitely reflected by their residuals, and $\xi_{k+1}$ plays a role whenever an approximate solution of the linear system is invoked. Ignoring or overlooking the role of $\xi_{k+1}$ is obviously incorrect. For example, Xue and Elman [21] have adapted the stopping criteria from [15]. Seemingly, the criterion does not involve $\xi_{k+1}$ explicitly but it requires that $p_{m}\left(\lambda_{1}-\theta_{k}\right)$ be reasonably small, say $10^{-3}$ to $10^{-2}$, where $p_{m}(z)$ is the associated residual polynomial of $m$-step MINRES for the linear system and $\theta_{k}$ is the Rayleigh quotient of the inexact RQI at step $k$. However, the size of $p_{m}\left(\lambda_{1}-\theta_{k}\right)$ is very closely related to $\xi_{k+1}$. Actually, it is easy to justify from the theory of MINRES that a small $\left|p_{m}\left(\lambda_{1}-\theta_{k}\right)\right|$ must mean a small $\xi_{k+1}$, and vice versa. Therefore, although $\xi_{k+1}$ is not necessarily small, its size does play a key role in its own intrinsic way in determining the cubic, quadratic and linear convergence rates of the inexact RQI. It is nontrivial and delicate for us to find out the correct quantitative role of $\xi_{k+1}$ and provide more insights into the convergence rates of the inexact RQI.

Before 22] was available in a technical report form in September 2010, the author of this paper in June 2009 gave a refined analysis on the inexact RQI with MINRES used for solving the linear systems, and the latest third version was available in June 2010 [9]. It is proved that the RQI with MINRES generally achieves the cubic asymptotic convergence whenever $\xi_{k+1}$ is not near one. The quadratic asymptotic convergence quantitatively requires $\xi_{k+1}=$ $1-O\left(\left\|r_{k}\right\|\right)$ (here we express it qualitatively), which is increasingly nearer to one as outer iterations proceeds and is much more relaxed than the existing ones in literature. Also, the linear convergence conditions are given that quantitatively require only $\xi_{k+1}=1-O\left(\left\|r_{k}\right\|^{2}\right)$, nearer to one than for the quadratic convergence. For the inexact RQI, to the author's best knowledge, there has been no result available on linear convergence. Besides [9], numerical experiments in [1, 22] have also confirmed such cubic asymptotic convergence.

As we have noticed, the basic condition $\xi_{k+1} \leq \xi<1$ is first assumed in all the above mentioned papers except [9]. This condition, though seemingly natural and necessary, may miss something essential and prevents us from getting better and more insightful results on the convergence of the inexact RQI. The author in [9] has revisited the convergence of the inexact RQI independent of iterative solvers and presents new general local convergence results. It is proved that the inexact RQI converges quadratically under a so-called uniform positiveness condition, which retains more information on inner solves and is fundamentally different from and weaker than the condition $\xi_{k+1} \leq \xi<1$. Although several results have been established for the inexact RQI with Lanczos in literature, one treats the residuals obtained by the Lanczos method as general ones and simply takes their norms in convergence analysis. Therefore, residual directions of inner iterations have not been reasonably exploited, and fundamental effects of residual directions on convergence have been completely overlooked. Based on the new general convergence results in [9], we first establish some attractive properties of the residuals obtained by the Lanczos method for the linear systems. By fully exploiting them, we then make a novel analysis and derive a number of insightful results that are not only stronger than but also fundamentally different from the ones available in literature. 
We should stress an important fact that each shifted inner linear system involved in the inexact RQI is typically Hermitian indefinite. For indefinite problems, it is well known that the Lanczos method typically behaves irregularly, that is, residual norms $\xi_{k+1}$ 's of the approximate solutions of the linear system can be large and even infinite for some Lanczos steps. For the inexact RQI with the unpreconditioned Lanczos, the most remarkable results we will prove are that the inexact RQI with Lanczos asymptotically converges quadratically provided that $\xi_{k+1} \leq \xi$ with a constant $\xi$ that is allowed to be bigger than one and the method is guaranteed to converge linearly provided that $\xi_{k+1}$ is bounded by not exceeding a moderate multiple of $\frac{1}{\left\|r_{k}\right\|}$, which means that we allow $\xi_{k+1} \gg 1$. The results have a strong impact on effective implementations of the method. Based the new theory, we design new stopping criteria for inner solves. To achieve the quadratic convergence, compared with prevailing implementations of the method, our new implementation can save the computational cost of solving the linear systems very significantly. Numerical experiments demonstrate that the new implementation is twice to four times and even more as fast as the prevailing implementations and the method converges smoothly and quickly for some problems even when $\xi_{k+1}$ is up to $10^{4} \sim 10^{7}$.

As byproducts, similar to that done in [9, 15, we establish lower bounds on the norms of approximate solutions $w_{k+1}$ of the linear systems obtained by the unpeconditioned Lanczos. We show that $\left\|w_{k+1}\right\|$ is always $O\left(\frac{1}{\left\|r_{k}\right\|^{2}}\right)$ no matter the inexact RQI with Lanczos converges cubically or quadratically. Therefore, it is distinctive that $\left\|w_{k+1}\right\|$ itself obtained by Lanczos cannot reveal the convergence behavior of the inexact RQI and cannot be used to design stopping criteria for inner iterations. Making use of these bounds, we present a simpler but weaker quadratic convergence result. As a global result, similar to that for the inexact RQI with MINRES [15] where it is shown that outer residual norms $\left\|r_{k}\right\|$ do not decrease monotonically any more for an arbitrary starting vector, we derive a relationship between $\left\|r_{k}\right\|$ and $\left\|r_{k+1}\right\|$, starting with an arbitrary vector instead of a reasonably good one. We will see that, unlike the exact RQI, the inexact RQI with Lanczos loses the residual monotonic decreasing property for an arbitrary starting vector. Therefore, for the convergence of the inexact RQI, it is only meaningful to speak of local rather than global convergence. That is, under the assumption that the current approximation has already a reasonable accuracy, one investigates how the next approximation better approaches the desired eigenvector. By convergence (rate), we always mean asymptotic convergence (rate).

We also extend our theory to the inexact RQI with a tuned preconditioned Lanczos method. This is a nontrivial task. We show that our main results in the unpreconditioned case can be extended to the tuned preconditioned case.

The paper is organized as follows. In Section 2, we review the inexact RQI and the new general convergence theory of [9] on the inexact RQI. In Section 3, we present convergence results on the inexact RQI with the unpreconditioned Lanczos Lanczos for solving inner linear systems. In Section 4, we extend the theory to the inexact RQI with a tuned preconditioned Lanczos method for solving inner linear systems. We perform numerical experiments to confirm our results in Section 5. Finally, we end up with some concluding remarks in Section 6,

Throughout the paper, denote by the superscript $*$ the conjugate transpose of a matrix or vector, by $\|\cdot\|$ the vector 2 -norm and the matrix spectral norm, and by $\lambda_{\min }, \lambda_{\max }$ the algebraically smallest and largest eigenvalues of $A$, respectively.

\section{The inexact RQI and general convergence theory}

RQI is a famous iterative algorithm and its locally cubic convergence for Hermitian problems is very attractive [13. It plays a crucial role in some practical effective algorithms, e.g., 
the QR algorithm, [8, 13. Assume that the unit length $u_{k}$ is already a reasonably good approximation to $x_{1}$. Then the Rayleigh quotient $\theta_{k}=u_{k}^{*} A u_{k}$ is a good approximation to $\lambda_{1}$ too. RQI [8, 13] computes a new approximation $u_{k+1}$ to $x_{1}$ by solving the shifted inner linear system

$$
\left(A-\theta_{k} I\right) w=u_{k}
$$

for $w_{k+1}$ and updating $u_{k+1}=w_{k+1} /\left\|w_{k+1}\right\|$ and iterates until convergence. It is known [1, 10, 13, that if

$$
\left|\lambda_{1}-\theta_{0}\right|<\frac{1}{2} \min _{j=2,3, \ldots, n}\left|\lambda_{1}-\lambda_{j}\right|
$$

then RQI asymptotically converges to $\lambda_{1}$ and $x_{1}$ cubically. So we can assume that the eigenvalues of $A$ are ordered as

$$
\left|\lambda_{1}-\theta_{k}\right|<\left|\lambda_{2}-\theta_{k}\right| \leq \cdots \leq\left|\lambda_{n}-\theta_{k}\right| \text { for all } k \text {. }
$$

With this ordering and noting that $\lambda_{\min } \leq \theta_{k} \leq \lambda_{\max }$, we have

$$
\left|\lambda_{1}-\theta_{k}\right|<\frac{1}{2}\left|\lambda-\lambda_{2}\right| \text {. }
$$

In the inexact RQI, (4) is solved by an iterative solver and an approximate solution $w_{k+1}$ satisfies

$$
\left(A-\theta_{k} I\right) w_{k+1}=u_{k}+\xi_{k+1} d_{k+1}, \quad u_{k+1}=w_{k+1} /\left\|w_{k+1}\right\|
$$

with $0<\xi_{k+1} \leq \xi$, where $\xi_{k+1} d_{k+1}$ with $\left\|d_{k+1}\right\|=1$ is the residual of $\left(A-\theta_{k} I\right) w=u_{k}$, $d_{k+1}$ is the residual direction vector and $\xi_{k+1}$ is the relative residual norm (inner tolerance) as $\left\|u_{k}\right\|=1$ and may change at every outer iteration $k$. This process is summarized as Algorithm 1. If $\xi_{k+1}=0$ for all $k$, Algorithm 1 becomes the exact RQI.

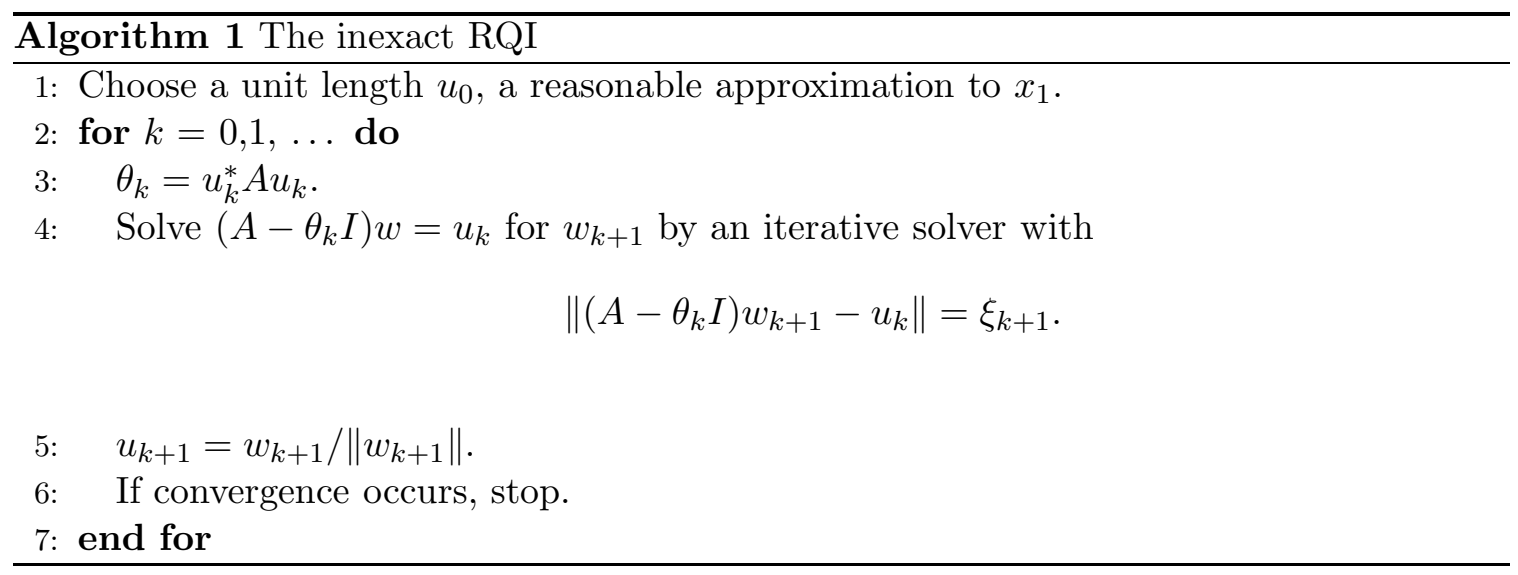

There are a number of general local quadratic convergence results in, e.g., 11, 15, 16, 19], which are all obtained by first requiring $\xi_{k+1} \leq \xi<1$. In [9], new local general convergence results have been proved under a new condition that is fundamentally different and can relax $\xi_{k+1}$ very much. To present the results, we decompose $u_{k}$ and $d_{k+1}$ into the orthogonal direct sums

$$
\begin{gathered}
u_{k}=x \cos \phi_{k}+e_{k} \sin \phi_{k}, \quad e_{k} \perp x, \\
d_{k+1}=x \cos \psi_{k}+f_{k} \sin \psi_{k}, \quad f_{k} \perp x
\end{gathered}
$$

with $\left\|e_{k}\right\|=\left\|f_{k}\right\|=1$ and $\phi_{k}=\angle\left(u_{k}, x\right), \psi_{k}=\angle\left(d_{k+1}, x\right)$. Here without loss of generality and for brevity of discussions, we suppose that $\phi_{k}$ is the acute angle between $u_{k}$ and $x_{1}$. 
Furthermore, we stress again that speaking of local convergence analysis naturally means that $\phi_{k}$ is already reasonably small, i.e., $\cos \phi_{k} \approx 1$ and $\sin \phi_{k} \approx 0$, and one then investigates how the next $u_{k+1}$ and $\theta_{k+1}$ better approximate $x_{1}$ and $\lambda_{1}$.

Given this, we should remind that $\cos \psi_{k}$ is either positive or negative depending on $d_{k+1}$. Note that (7) can be written as

$$
\left(A-\theta_{k} I\right) w_{k+1}=\left(\cos \phi_{k}+\xi_{k+1} \cos \psi_{k}\right) x+\left(e_{k} \sin \phi_{k}+\xi_{k+1} f_{k} \sin \psi_{k}\right) .
$$

Inverting $A-\theta_{k} I$ gives

$$
w_{k+1}=\left(\lambda_{1}-\theta_{k}\right)^{-1}\left(\cos \phi_{k}+\xi_{k+1} \cos \psi_{k}\right) x+\left(A-\theta_{k} I\right)^{-1}\left(e_{k} \sin \phi_{k}+\xi_{k+1} f_{k} \sin \psi_{k}\right) .
$$

Define $\left\|r_{k}\right\|=\left\|\left(A-\theta_{k} I\right) u_{k}\right\|$. Then by (6) we get $\left|\lambda_{2}-\theta_{k}\right|>\frac{\left|\lambda_{2}-\lambda_{1}\right|}{2}$. It is known from [13, Theorem 11.7.1] that

$$
\frac{\left\|r_{k}\right\|}{\lambda_{\max }-\lambda_{\min }} \leq \sin \phi_{k} \leq \frac{2\left\|r_{k}\right\|}{\left|\lambda_{2}-\lambda_{1}\right|}
$$

We comment that $\lambda_{\max }-\lambda_{\min }$ is the spectrum spread of $A$ and $\left|\lambda_{2}-\lambda_{1}\right|$ is the gap or separation of $\lambda_{1}$ and the other eigenvalues of $A$.

Throughout the paper, we define

$$
\beta=\frac{\lambda_{\max }-\lambda_{\min }}{\left|\lambda_{2}-\lambda_{1}\right|}
$$

Theorem 1. 9] If the uniform positiveness condition

$$
\left|\cos \phi_{k}+\xi_{k+1} \cos \psi_{k}\right| \geq c
$$

is satisfied with a moderate constant $c>0$ independent of $k$, then

$$
\begin{aligned}
\tan \phi_{k+1} & \leq 2 \beta \frac{\sin \phi_{k}+\xi_{k+1} \sin \psi_{k}}{\left|\cos \phi_{k}+\xi_{k+1} \cos \psi_{k}\right|} \sin ^{2} \phi_{k} \\
& \leq \frac{2 \beta \xi_{k+1}}{c} \sin ^{2} \phi_{k}+O\left(\sin ^{3} \phi_{k}\right)
\end{aligned}
$$

that is, the inexact RQI asymptotically converges quadratically provided that (14) is satisfied and $\xi_{k+1}$ is uniformly bounded by some moderate constant.

It can be found in [9] that the proof of (15) is elementary and easy to follow. Combining (15) and (14), it is direct to get (16).

Theorem 2. [9] If the uniform positiveness condition (14) holds, then

$$
\left\|r_{k+1}\right\| \leq \frac{8 \beta^{2} \xi_{k+1}}{c\left|\lambda_{2}-\lambda_{1}\right|}\left\|r_{k}\right\|^{2}+O\left(\left\|r_{k}\right\|^{3}\right)
$$

We make some comments on the above two theorems.

Remark 1. They illustrate that it is the size of $\left|\cos \phi_{k}+\xi_{k+1} \cos \psi_{k}\right|$ other than $\xi_{k+1} \leq \xi<1$ that is critical in convergence.

Remark 2. If $\xi_{k+1}=0$ for all $k$, then the inexact RQI reduces to the exact RQI and Theorems 1 2] show the cubic convergence: $\tan \phi_{k+1} \leq \frac{2 \beta}{\cos \phi_{k}} \sin ^{3} \phi_{k}$ and $\left\|r_{k+1}\right\|=O\left(\left\|r_{k}\right\|^{3}\right)$. If the linear systems are solved with decreasing tolerance $\xi_{k+1}=O\left(\sin \phi_{k}\right)=O\left(\left\|r_{k}\right\|\right)$, then we have the cubic convergence: $\tan \phi_{k+1}=O\left(\sin ^{3} \phi_{k}\right)$ and $\left\|r_{k+1}\right\|=O\left(\left\|r_{k}\right\|^{3}\right)$. 
Remark 3. If $\cos \psi_{k}$ is positive, the uniform positiveness condition holds for any uniformly bounded $\xi_{k+1} \leq \xi$ with $\xi$ a moderate constant. So we may have $\xi \geq 1$. If $\cos \psi_{k}$ is negative, the uniform positiveness condition $\left|\cos \phi_{k}+\xi_{k+1} \cos \psi_{k}\right| \geq c$ means that

$$
\xi_{k+1} \leq \frac{c-\cos \phi_{k}}{\cos \psi_{k}}
$$

if $\cos \phi_{k}+\xi_{k+1} \cos \psi_{k} \geq c$ and

$$
\xi_{k+1} \geq \frac{c+\cos \phi_{k}}{-\cos \psi_{k}}
$$

if $-\cos \phi_{k}-\xi_{k+1} \cos \psi_{k} \geq c$. So the size of $\xi_{k+1}$ critically depends on that of $\cos \psi_{k}$, and for a given $c$ we may have $\xi_{k+1} \approx 1$ and even $\xi_{k+1}>1$. Obviously, without the information on $\cos \psi_{k}$, it would be impossible to access or estimate $\xi_{k+1}$. As a general convergence result, however, its significance and importance consist in that it fully exploits a crucial quantity $\cos \psi_{k}$ to relax $\xi_{k+1}$ as much as possible and meanwhile preserves the same convergence rate of outer iteration. As a result, the condition $\xi_{k+1} \leq \xi<1$ with constant $\xi$ not near one may be stringent and unnecessary for the quadratic convergence of the inexact RQI, independent of iterative solvers for inner linear systems.

Remark 4. Keep in mind a basic fact that the bigger $\xi_{k+1}$ is, the less costly a chosen inner iterative solver is. The new conditions on $\xi_{k+1}$ derived from the uniform positiveness condition have a strong impact on effective implementations of the inexact RQI since we must stop a certain iterative solver, e.g., the very popular MINRES method and the Lanczos method for solving $\left(A-\theta_{k} I\right) w=u_{k}$ at right moment. It appears that $\cos \psi_{k}$ is critically iterative solver dependent. For Lanczos, $\cos \psi_{k}$ has some very attractive properties. Making use of them, we can precisely determine bounds for $\xi_{k+1}$ in Section 3, which are much more relaxed than those in literature. For the convergence of the inexact RQI with MINRES, we refer to [9] for the properties of $\cos \psi_{k}$ and their effects on $\xi_{k+1}$.

\section{Convergence of the inexact RQI with the unpreconditioned Lanczos}

The previous results and discussions are for general purpose, independent of iterative solvers for $\left(A-\theta_{k} I\right) w=u_{k}$. Since we have $\lambda_{\min } \leq \theta_{k} \leq \lambda_{\max }$, the matrix $A-\theta_{k} I$ is Hermitian indefinite when $\theta_{k} \neq \lambda_{1}$. The Lanczos method is a popular Krylov subspace iterative solver for Hermitian linear systems [14. The method nicely fits into the inexact RQI.

We briefly review the Lanczos method for solving (4). At outer iteration $k$, taking the starting vector $v_{1}$ to be $u_{k}$, the $m$-step Lanczos process on $A-\theta_{k} I$ can be written as

$$
\left(A-\theta_{k} I\right) V_{m}=V_{m} T_{m}+t_{m+1 m} v_{m+1} e_{m}^{*},
$$

where the columns of $V_{m}=\left(v_{1}, \ldots, v_{m}\right)$ form an orthonormal basis of the Krylov subspace $\mathcal{K}_{m}\left(A-\theta_{k} I, u_{k}\right)=\mathcal{K}_{m}\left(A, u_{k}\right)$ and $T_{m}=\left(t_{i j}\right)=V_{m}^{*}\left(A-\theta_{k} I\right) V_{m}$ is an $m \times m$ Hermitian tridiagonal matrix [13, 14].

The Lanczos method [8, 11, 14] is a Galerkin projection method and requires the residual $\xi_{k+1} d_{k+1}$ to be orthogonal to the search subspace. With the zero vector as an initial guess to the solution of $\left(A-\theta_{k} I\right) w=u_{k}$, the Galerkin condition means $\xi_{k+1} d_{k+1} \perp \mathcal{K}_{m}\left(A, u_{k}\right)$. Specially, $\xi_{k+1} d_{k+1} \perp u_{k} \in \mathcal{K}_{m}\left(A, u_{k}\right)$. The method extracts the approximate solution $w_{k+1}=V_{m} \hat{y}$ to $\left(A-\theta_{k} I\right) w=u_{k}$ from $\mathcal{K}_{m}\left(A, u_{k}\right)$, where $\hat{y}$ is the solution of the Hermitian tridiagonal linear system $T_{m} y=e_{1}$ with $e_{1}$ being the first coordinate vector of dimension $m$. It is worth noting that we should naturally take $m>1$; otherwise we would have $\mathcal{K}_{1}\left(A, u_{k}\right)=\operatorname{span}\left\{u_{k}\right\}$ and $T_{1}=0$, so that the Lanczos method would break down and 
$w_{k+1}$ would not exist. The algorithm SYMMLQ is a very effective implementation of the Lanczos method [8, 12].

For Hermitian positive definite linear systems, the Lanczos method is mathematically equivalent to the conjugate gradient method and has the optimality that the error of the approximate solution is minimal with respect to the energy norm over the given Krylov subspace [14. For Hermitian indefinite linear systems, the method does not have any kind of optimality. For our case, the linear system $\left(A-\theta_{k} I\right) w=u_{k}$ is not only indefinite but also increasingly ill conditioned as $\theta_{k} \rightarrow \lambda_{1}$. The indefinite system is typically ill conditioned and can be (nearly) singular, so that the Lanczos method may converge slowly and irregularly and $\xi_{k+1}$ can be typically big for $m$ small. For more details, we refer to [11, 14].

We will present convergence results on the inexact RQI with Lanczos. First of all, we establish the following results, which will play a key role in the later analysis.

Theorem 3. It holds that

$$
\left|\cos \psi_{k}\right| \leq \tan \phi_{k}
$$

and asymptotically

$$
\sin \psi_{k} \geq 1-\frac{1}{2} \sin ^{2} \phi_{k}
$$

by ignoring the higher order term $O\left(\sin ^{4} \phi_{k}\right)$.

Proof. Recall that its residual $\xi_{k+1} d_{k+1}$ obtained by the Lanczos method satisfies $\xi_{k+1} d_{k+1} \perp$ $\mathcal{K}_{m}\left(A, u_{k}\right)$. So, we specially have $d_{k+1} \perp u_{k}$. Therefore, from (8) and (9) we get

$$
\cos \phi_{k} \cos \psi_{k}+e_{k}^{*} f_{k} \sin \phi_{k} \sin \psi_{k}=0,
$$

which means

$$
\frac{\left|\cos \psi_{k}\right|}{\sin \psi_{k}}=\left|e_{k}^{*} f_{k} \tan \phi_{k}\right| \leq \tan \phi_{k}
$$

From the above it follows that (19) holds. By the Taylor expansion, from (20) we get

$$
\sin \psi_{k}=\sqrt{1-\cos ^{2} \psi_{k}}=1-\frac{1}{2}\left|\cos ^{2} \psi_{k}\right|+O\left(\cos ^{4} \psi_{k}\right) \geq 1-\frac{1}{2} \tan ^{2} \phi_{k}=1-\frac{1}{2} \sin ^{2} \phi_{k}
$$

by dropping the higher order term $O\left(\sin ^{4} \phi_{k}\right)$.

Combining this theorem with (15) of Theorem 1, we can establish one of our main results for the inexact RQI with Lanczos.

Theorem 4. Let $\xi$ be a constant such that $\xi_{k+1} \leq \xi$ and satisfy

$$
\xi \sin \phi_{k} \leq \alpha<1
$$

with $\alpha$ a given constant not near one. Then the uniform positiveness condition (14) holds and the inexact RQI with Lanczos asymptotically converges quadratically:

$$
\tan \phi_{k+1} \leq \frac{2 \beta \xi}{1-\alpha} \sin ^{2} \phi_{k}
$$

It asymptotically converges cubically if

$$
\xi_{k+1}=O\left(\sin \phi_{k}\right)
$$

it converges at linear factor $\gamma<1$ :

$$
\tan \phi_{k+1} \leq \gamma \sin \phi_{k}
$$

if

$$
\xi_{k+1} \leq \frac{\gamma-2 \beta \sin ^{2} \phi_{k}}{(2 \beta+\gamma) \sin \phi_{k}}<\frac{\gamma}{(2 \beta+\gamma) \sin \phi_{k}}
$$


Proof. By (20), we get

$$
\sin \phi_{k}+\xi_{k+1} \sin \psi_{k} \leq \sin \phi_{k}+\xi_{k+1} \leq \sin \phi_{k}+\xi
$$

On the other hand, we get from (19)

$$
\begin{aligned}
\left|\cos \phi_{k}+\xi_{k+1} \cos \psi_{k}\right| & \geq\left|\cos \phi_{k}-\xi_{k+1}\right| \cos \psi_{k}|| \\
& \geq\left|1-\frac{1}{2} \sin ^{2} \phi_{k}+O\left(\sin ^{4} \phi_{k}\right)-\xi_{k+1} \tan \phi_{k}\right| \\
& =\left|1-\xi_{k+1} \sin \phi_{k}+O\left(\sin ^{2} \phi_{k}\right)\right| \\
& \geq 1-\xi \sin \phi_{k} \\
& \geq 1-\alpha,
\end{aligned}
$$

by dropping the higher order term $O\left(\sin ^{2} \phi_{k}\right)$. So the uniform positiveness condition (14) holds with $c=1-\alpha$. We then derive from (15) that

$$
\begin{aligned}
\tan \phi_{k+1} & \leq 2 \beta \frac{\sin \phi_{k}+\xi_{k+1} \sin \psi_{k}}{\left|\cos \phi_{k}+\xi_{k+1} \cos \psi_{k}\right|} \sin ^{2} \phi_{k} \\
& \leq 2 \beta \frac{\left(\sin \phi_{k}+\xi_{k+1}\right)}{\left|1-\xi_{k+1} \sin \phi_{k}\right|} \sin ^{2} \phi_{k} \\
& \leq 2 \beta \frac{\left(\sin \phi_{k}+\xi\right)}{1-\alpha} \sin ^{2} \phi_{k} \\
& \leq 2 \beta \frac{\xi}{1-\alpha} \sin ^{2} \phi_{k}+O\left(\sin ^{3} \phi_{k}\right)
\end{aligned}
$$

which is just (22) by ignoring $O\left(\sin ^{3} \phi_{k}\right)$.

The cubic asymptotic convergence is direct from (27) if $\xi_{k+1}=O\left(\sin \phi_{k}\right)$.

It follows from (27) that the inexact RQI with Lanczos converges at linear factor $\gamma$ at least if for all $k$ it holds that $\xi_{k+1} \sin \phi_{k}<1$ and

$$
2 \beta \frac{\left(\sin \phi_{k}+\xi_{k+1}\right) \sin \phi_{k}}{1-\xi_{k+1} \sin \phi_{k}} \leq \gamma<1
$$

from which we get condition (25) by manipulation.

Theorem 4 presents the conditions on cubic, quadratic and linear convergence in terms of an a priori uncomputable $\sin \phi_{k}$. We next give their alternatives in terms of the computable $\left\|r_{k}\right\|$, so that they are of practical value as much as possible and can be used to control the inner tolerance to achieve a desired convergence rate.

Theorem 5. Let $\xi$ be a constant such that $\xi_{k+1} \leq \xi$ for all $k$ and satisfy

$$
\frac{2 \xi\left\|r_{k}\right\|}{\left|\lambda_{2}-\lambda_{1}\right|} \leq \alpha<1
$$

with $\alpha$ a given constant not near one. The uniform positiveness condition holds and the inexact RQI with Lanczos asymptotically converges quadratically:

$$
\begin{aligned}
\left\|r_{k+1}\right\| & \leq \frac{8 \beta^{2}\left(2\left\|r_{k}\right\|+\xi_{k+1}\left|\lambda_{2}-\lambda_{1}\right|\right)}{\left|\lambda_{2}-\lambda\right|\left(\left|\lambda_{2}-\lambda_{1}\right|-2 \xi_{k+1}\left\|r_{k}\right\|\right)}\left\|r_{k}\right\|^{2} \\
& \leq \frac{8 \beta^{2}\left(2\left\|r_{k}\right\|+\xi\left|\lambda_{2}-\lambda_{1}\right|\right)}{\left(\lambda_{2}-\lambda_{1}\right)^{2}(1-\alpha)}\left\|r_{k}\right\|^{2}
\end{aligned}
$$


It asymptotically converges cubically if

$$
\xi_{k+1}=O\left(\left\|r_{k}\right\|\right)
$$

it converges at linear factor $\gamma<1$ :

$$
\left\|r_{k+1}\right\| \leq \gamma\left\|r_{k}\right\|
$$

if

$$
\xi_{k+1} \leq \frac{\gamma\left(\lambda_{2}-\lambda_{1}\right)^{2}-16 \beta^{2}\left\|r_{k}\right\|^{2}}{2\left|\lambda_{2}-\lambda_{1}\right|\left(4 \beta^{2}+\gamma\right)\left\|r_{k}\right\|}<\frac{\gamma\left|\lambda_{2}-\lambda_{1}\right|}{\left(8 \beta^{2}+2 \gamma\right)\left\|r_{k}\right\|}
$$

Proof. Making use of (12) gives

$$
\begin{gathered}
\frac{\left\|r_{k+1}\right\|}{\lambda_{\max }-\lambda_{\min }} \leq \sin \phi_{k+1} \leq \tan \phi_{k+1} \\
1-\xi_{k+1} \sin \phi_{k} \geq 1-\xi_{k+1} \frac{2\left\|r_{k}\right\|}{\left|\lambda_{2}-\lambda_{1}\right|} \geq 1-\frac{2 \xi\left\|r_{k}\right\|}{\left|\lambda_{2}-\lambda_{1}\right|} \geq 1-\alpha>0
\end{gathered}
$$

and

$$
\sin \phi_{k}+\xi_{k+1} \leq \frac{2\left\|r_{k}\right\|}{\left|\lambda_{2}-\lambda_{1}\right|}+\xi_{k+1}
$$

Substituting the above relations into (27) and (22) establishes (29) and (30), respectively. It is clear from (29) that the inexact RQI with Lanczos asymptotically converges cubically once $\xi_{k+1}=O\left(\left\|r_{k}\right\|\right)$.

In order to make $\left\|r_{k}\right\|$ linearly converge to zero monotonically, from (29) we simply set

$$
\frac{8\left(\lambda_{n}-\lambda\right)^{2}}{\left|\lambda_{2}-\lambda_{1}\right|^{3}} \frac{2\left\|r_{k}\right\|+\xi_{k+1}\left|\lambda_{2}-\lambda_{1}\right|}{\left|\lambda_{2}-\lambda_{1}\right|-2 \xi_{k+1} \| r_{k} \mid}\left\|r_{k}\right\| \leq \gamma<1 .
$$

Solving it for $\xi_{k+1}$ gives

$$
\xi_{k+1} \leq \frac{\gamma\left(\lambda_{2}-\lambda_{1}\right)^{2}-16 \beta^{2}\left\|r_{k}\right\|^{2}}{2\left|\lambda_{2}-\lambda_{1}\right|\left(4 \beta^{2}+\gamma\right)\left\|r_{k}\right\|}<\frac{\gamma\left|\lambda_{2}-\lambda_{1}\right|}{\left(8 \beta^{2}+2 \gamma\right)\left\|r_{k}\right\|}
$$

We make some comments on Theorems 45 ,

Remark 1. The quadratic asymptotic convergence condition (21) indicates that $\xi>1$ is allowed as $\xi \leq \frac{\alpha}{\sin \phi_{k}}$ and $\sin \phi_{k}$ is supposed to small. For a given reasonably good starting vector $u_{0}$, if both the global convergence and quadratic asymptotic convergence are required, then only (21) may not be sufficient. It is seen from (22) that if $\xi$ satisfies

$$
\tan \phi_{1} \leq \frac{2 \beta \xi}{1-\alpha} \sin ^{2} \phi_{0}<\tan \phi_{0}
$$

then $\tan \phi_{k}$ decreases from the beginning of outer iteration. From (34) we find

$$
\xi<\frac{1-\alpha}{\beta \sin 2 \phi_{0}} .
$$

Combining it with the requirement $\xi \leq \frac{\alpha}{\sin \phi_{0}}$, we get

$$
\xi<\min \left\{\frac{\alpha}{\sin \phi_{0}}, \frac{1-\alpha}{\beta \sin 2 \phi_{0}}\right\}
$$


With such $\xi$, the inexact RQI with Lanczos achieves the quadratic asymptotic convergence. The upper bound depends on $\beta$, which measures the conditioning of $x_{1}$. We see that $\xi$ may be bigger than one as $\sin \phi_{0}$ is reasonably small. So the old requirement $\xi_{k+1} \leq \xi<1$ is stringent and not necessary for the quadratic asymptotic convergence. Similar comments can be made on (28) in Theorem 5 as well. We should point out that the above bound for $\xi$ is conservative, and numerical experiments will demonstrate that the inexact RQI with Lanczos works well and achieves the quadratic convergence when $\xi$ exceeds bound (35).

Remark 2. Conditions (25) and (33) for linear convergence show that $\xi_{k+1}$ can be as big as $O\left(\frac{1}{\sin \phi_{k}}\right)$ and $O\left(\frac{1}{\left\|r_{k}\right\|}\right)$ as outer iterations proceed. More precisely, (25) indicates that the inexact RQI with Lanczos still converges linearly even if $\xi_{k+1}$ is as big as $O\left(\frac{1}{\sin \phi_{k}}\right)$ with the order constant $\frac{\gamma}{2 \beta+\gamma}$ smaller than one.

As done in [9, 15], we now estimate $\left\|w_{k+1}\right\|$ in (7) obtained by Lanczos. Note that the exact solution of $\left(A-\theta_{k} I\right) w=u_{k}$ is $w_{k+1}=\left(A-\theta_{k} I\right)^{-1} u_{k}$. Therefore, setting $\xi_{k+1}=0$ in (11), we have

$$
\begin{aligned}
\left\|w_{k+1}\right\| & =\frac{\cos \phi_{k}}{\left|\theta_{k}-\lambda_{1}\right|}+O\left(\sin \phi_{k}\right) \\
& \approx \frac{1}{\left|\theta_{k}-\lambda_{1}\right|}=\left\|\left(A-\theta_{k} I\right)^{-1}\right\| \\
& =O\left(\frac{1}{\sin ^{2} \phi_{k}}\right)=O\left(\frac{1}{\left\|r_{k}\right\|^{2}}\right),
\end{aligned}
$$

the last equality being from (12). From (111) and (12), we also see that these estimates hold for $\xi_{k+1}=O\left(\sin \phi_{k}\right)=O\left(\left\|r_{k}\right\|\right)$. So $\left\|w_{k+1}\right\|$ is also $O\left(\frac{1}{\left\|r_{k}\right\|^{2}}\right)$ when the inexact RQI with Lanczos converges cubically. Next we derive quantitative estimates on $\left\|w_{k+1}\right\|$ under more general conditions, and by the estimates we establish a new quadratic convergence result.

Theorem 6. Let $\xi$ be a constant such that $\xi_{k+1} \leq \xi$ and satisfy $\xi \sin \phi_{k} \leq \alpha<1$ with $\alpha$ a given constant not near one. Then we have

$$
\begin{aligned}
\left\|w_{k+1}\right\| & \geq \frac{(1-\alpha)\left|\lambda_{2}-\lambda_{1}\right|}{4 \beta\left\|r_{k}\right\|^{2}} \\
\left\|r_{k+1}\right\| & \leq \frac{\sqrt{1+\xi^{2}}}{\left\|w_{k+1}\right\|}, \\
\left\|r_{k+1}\right\| & \leq \frac{4 \beta \sqrt{1+\xi^{2}}}{\left|\lambda_{2}-\lambda_{1}\right|(1-\alpha)}\left\|r_{k}\right\|^{2},
\end{aligned}
$$

where (36) and (38) hold asymptotically and (37) holds exactly.

Proof. As $\xi_{k+1} d_{k+1} \perp u_{k}$ and $\left(A-\theta_{k} I\right) w_{k+1}=u_{k}+\xi_{k+1} d_{k+1}$, we have

$$
\left\|\left(A-\theta_{k} I\right) w_{k+1}\right\|^{2}=\left\|u_{k}\right\|^{2}+\left\|\xi_{k+1} d_{k+1}\right\|^{2}=1+\xi_{k+1}^{2} .
$$

So we get from $u_{k+1}=w_{k+1} /\left\|w_{k+1}\right\|$ that

$$
\left\|\left(A-\theta_{k} I\right) u_{k+1}\right\|=\frac{\sqrt{1+\xi_{k+1}^{2}}}{\left\|w_{k+1}\right\|} \leq \frac{\sqrt{1+\xi^{2}}}{\left\|w_{k+1}\right\|} .
$$

By the optimality of Rayleigh quotient we obtain

$$
\left\|r_{k+1}\right\|=\left\|\left(A-\theta_{k+1} I\right) u_{k+1}\right\| \leq\left\|\left(A-\theta_{k} I\right) u_{k+1}\right\|=\frac{\sqrt{1+\xi_{k+1}^{2}}}{\left\|w_{k+1}\right\|} \leq \frac{\sqrt{1+\xi^{2}}}{\left\|w_{k+1}\right\|},
$$


which shows (37). It is easy to verify (cf. [13, p. 77]) that

$$
\left|\lambda_{2}-\lambda_{1}\right| \sin ^{2} \phi_{k} \leq\left|\lambda_{1}-\theta_{k}\right| \leq\left(\lambda_{\max }-\lambda_{\min }\right) \sin ^{2} \phi_{k} .
$$

By using (11), (26), (41) and (12) in turn, we obtain

$$
\begin{aligned}
\left\|w_{k+1}\right\| & \geq \frac{\left|\cos \phi_{k}+\xi_{k+1} \cos \psi_{k}\right|}{\left|\theta_{k}-\lambda_{1}\right|} \\
& \geq \frac{\left|1-\xi_{k+1} \sin \phi_{k}\right|}{\left|\theta_{k}-\lambda_{1}\right|} \\
& \geq \frac{1-\alpha}{\left|\theta_{k}-\lambda_{1}\right|} \\
& \geq \frac{1-\alpha}{\left(\lambda_{\max }-\lambda_{\min }\right) \sin ^{2} \phi_{k}} \\
& \geq \frac{(1-\alpha)\left|\lambda_{2}-\lambda_{1}\right|}{4 \beta\left\|r_{k}\right\|^{2}} \\
& \geq \frac{(1-\alpha)\left|\lambda_{2}-\lambda_{1}\right|}{4 \beta\left\|r_{k}\right\|^{2}},
\end{aligned}
$$

which proves (36). Substituting (36) into (37) establishes (38).

(38) indicates that the inexact RQI with Lanczos converges quadratically if $\alpha$ is not near one. By combining this theorem with Theorems 445. (36) shows that $\left\|w_{k+1}\right\|$ is always no less than $O\left(\frac{1}{\left\|r_{k}\right\|^{2}}\right)$ when the inexact RQI with Lanczos converges quadratically provided that $\alpha$ is not near one. Noting that the inexact RQI with Lanczos for $\xi_{k+1}=$ $O\left(\sin \phi_{k}\right)=O\left(\left\|r_{k}\right\|\right)$ converges cubically and $\left\|w_{k+1}\right\|$ is also $O\left(\frac{1}{\left\|r_{k}\right\|^{2}}\right)$ (cf. the comments before Theorem (6), this illustrates that the size of $\left\|w_{k+1}\right\|$ itself cannot reveal cubic and quadratic convergence rates of the inexact RQI with Lanczos. Furthermore, we cannot recover the cubic convergence of the exact RQI and the inexact RQI when $\xi_{k+1}=\xi=0$ and $\xi_{k+1}=\xi=O\left(\sin \phi_{k}\right)=O\left(\left\|r_{k}\right\|\right)$, respectively. So (38) is weaker than Theorems 44 5 , This is because $\left\|r_{k+1}\right\| \leq\left\|\left(A-\theta_{k} I\right) u_{k+1}\right\|$ is not sharp in the proof.

So far, all the convergence results are local, that is, they care how the exact and inexact RQI behaves only from the current outer iteration to the next one, assuming that current $\left(\theta_{k}, u_{k}\right)$ is already a reasonably good approximation to $(\lambda, x)$. As is well known, one of the important properties of the exact RQI is its global residual monotonic decreasing property, i.e., $\left\|r_{k+1}\right\| \leq\left\|r_{k}\right\|$, for any (poor) starting vector $u_{0}$; see Theorem 4.8 .1 of [13, p. 79]. We now present a global property to the inexact RQI with Lanczos.

Theorem 7. For the inexact RQI with Lanczos starting with any starting vector $u_{0}$, we have

$$
\left\|r_{k+1}\right\| \leq \sqrt{1+\xi_{k+1}^{2}}\left\|r_{k}\right\|, k=0,1, \ldots
$$

Proof. From (7), we have

$$
w_{k+1}=\left(A-\theta_{k} I\right)^{-1}\left(u_{k}+\xi_{k+1} d_{k+1}\right) .
$$

Again, note that for the Lanczos method its residual $\xi_{k+1} d_{k+1}$ satisfies $\xi_{k+1} d_{k+1}^{*} u_{k}=0$. 
Then from (40) and the Cauchy-Schwarz inequality, we get

$$
\begin{aligned}
\frac{\left\|r_{k+1}\right\|}{\left\|r_{k}\right\|} & \leq \frac{\left\|\left(A-\theta_{k} I\right) u_{k+1}\right\|}{\left\|r_{k}\right\|}=\frac{\sqrt{1+\xi_{k+1}^{2}}}{\left\|w_{k+1}\right\|\left\|r_{k}\right\|} \\
& =\frac{\sqrt{1+\xi_{k+1}^{2}}}{\left\|\left(A-\theta_{k} I\right)^{-1}\left(u_{k}+\xi_{k+1} d_{k+1}\right)\right\|\left\|\left(A-\theta_{k} I\right) u_{k}\right\|} \\
& \leq \frac{\sqrt{1+\xi_{k+1}^{2}}}{\left|\left(u_{k}+\xi_{k+1} d_{k+1}\right)^{*}\left(A-\theta_{k} I\right)^{-1}\left(A-\theta_{k} I\right) u_{k}\right|} \\
& =\frac{\sqrt{1+\xi_{k+1}^{2}}}{\left|1+\xi_{k+1} d_{k+1}^{*} u_{k}\right|}=\sqrt{1+\xi_{k+1}^{2}},
\end{aligned}
$$

which proves (42).

This theorem shows that, unlike the exact RQI, $\left\|r_{k}\right\|$ obtained by the inexact RQI with Lanczos is not monotonic decreasing in the global sense for an arbitrary starting vector $u_{0}$. This is similar to the inexact RQI with MINRES, where Simoncini and Eldén [15] have derived a similar relationship between $\left\|r_{k+1}\right\|$ and $\left\|r_{k}\right\|$, showing that residuals obtained by the inexact RQI with MINRES lose the monotonic decreasing property that the exact RQI possesses; see Theorem 5.4 of [15]. Therefore, as far as global convergence is concerned, the inexact RQI has a very essential difference from the exact RQI, the former cannot guarantee its convergence while the latter almost always converges for an arbitrary starting vector [13]. For the inexact RQI, we can only expect its local convergence starting with a reasonably good starting vector. We have made numerical experiments on some matrices for some starting $u_{0}$ 's generated randomly and found that it is indeed the case for the inexact RQI with Lanczos or MINRES.

\section{Convergence of the inexact RQI with a tuned precondi- tioned Lanczos}

We have found that for a given $\xi$ satisfying our convergence conditions, we may still need many inner iteration steps at each outer iteration. This is especially true for difficult problems, i.e., big $\beta$ 's, or for computing an interior eigenvalue $\lambda_{1}$ since it leads to a highly Hermitian indefinite matrix $\left(A-\theta_{k} I\right)$ at each outer iteration. So, in order to improve the overall performance, preconditioning is generally necessary to speed up the Lanczos method. Some preconditioning techniques have been proposed in e.g., [1, 15]. In the unpreconditioned case, the right-hand side $u_{k}$ of (44) is rich in the direction of the desired $x_{1}$. We can benefit much from this property when solving the linear system. Actually, if the right-hand side is an eigenvector of the coefficient matrix, Krylov subspace type methods will find the exact solution in one step. However, a usual preconditioner loses this important property, so that inner iteration steps may not be reduced [1, 4, 7]. A preconditioner with tuning is necessary to recover this property and meanwhile attempts to improve the conditioning of the preconditioned system, so that considerable improvement over a usual preconditioner is possible [4, 7, 21]. In what follows we show how to extend our previous theory to the inexact RQI with a tuned preconditioned Lanczos.

Let $Q=L L^{*}$ be a Cholesky factorization of some Hermitian positive definite matrix which is an approximation to $A-\theta_{k} I$ in some sense [1, 7, 21. A tuned preconditioner 
$\mathcal{Q}=\mathcal{L} \mathcal{L}^{*}$ can be constructed by adding a rank-1 or rank-2 modification to $Q$, so that

$$
\mathcal{Q} u_{k}=A u_{k}
$$

see [4, 7, 21] for details. Using the tuned preconditioner $\mathcal{Q}$, the shifted inner linear system (4) is equivalently transformed to the preconditioned one

$$
B \hat{w}=\mathcal{L}^{-1}\left(A-\theta_{k} I\right) \mathcal{L}^{-*} \hat{w}=\mathcal{L}^{-1} u_{k}
$$

with the original $w=\mathcal{L}^{-*} \hat{w}$. Once the Lanczos method is used to solve it, we are led to the inexact RQI with a tuned preconditioned Lanczos. A power of the tuned preconditioner $\mathcal{Q}$ is that the right-hand side $\mathcal{L}^{-1} u_{k}$ is rich in the eigenvector of $B$ associated with its smallest eigenvalue and has the same quality as $u_{k}$ as an approximation to the eigenvector $x_{1}$ of $A$, while for the usual preconditioner $Q$ the right-hand side $L^{-1} u_{k}$ does not possess this property.

Take the zero vector as an initial guess to the solution of (44) and let $\hat{w}_{k+1}$ be the approximate solution obtained by the $m$-step Lanczos method applied to it. Then we have

$$
\mathcal{L}^{-1}\left(A-\theta_{k} I\right) \mathcal{L}^{-*} \hat{w}_{k+1}=\mathcal{L}^{-1} u_{k}+\hat{\xi}_{k} \hat{d}_{k+1},
$$

where $\hat{w}_{k+1} \in \mathcal{K}_{m}\left(B, \mathcal{L}^{-1} u_{k}\right), \hat{\xi}_{k+1} \hat{d}_{k+1}$ with $\left\|\hat{d}_{k+1}\right\|=1$ is the residual and $\hat{d}_{k+1}$ is the residual direction vector. Keep in mind that $w_{k+1}=\mathcal{L}^{-*} \hat{w}_{k+1}$. We then get

$$
\left(A-\theta_{k} I\right) w_{k+1}=u_{k}+\hat{\xi}_{k+1} \mathcal{L} \hat{d}_{k+1}=u_{k}+\hat{\xi}_{k+1}\left\|\mathcal{L} \hat{d}_{k+1}\right\| \frac{\mathcal{L} \hat{d}_{k+1}}{\left\|\mathcal{L} \hat{d}_{k+1}\right\|} .
$$

So $\xi_{k+1}$ and $d_{k+1}$ in (7) are $\hat{\xi}_{k+1}\left\|\mathcal{L} \hat{d}_{k+1}\right\|$ and $\frac{\mathcal{L} \hat{d}_{k+1}}{\left\|\mathcal{L} \hat{d}_{k+1}\right\|}$, respectively. Hence our general Theorems 112 apply and are not repeated here.

An extension of Theorem 3 to the preconditioned case is nontrivial and needs more work. Let $\left(\mu_{i}, y_{i}\right), i=1,2, \ldots, n$ be the eigenpairs of $B$ with

$$
\left|\mu_{1}\right|<\left|\mu_{2}\right| \leq \cdots \leq\left|\mu_{n}\right|
$$

Define $\hat{u}_{k}=\mathcal{L}^{-1} u_{k} /\left\|\mathcal{L}^{-1} u_{k}\right\|$. Similar to (8) and (9), let

$$
\begin{aligned}
\hat{u}_{k} & =y_{1} \cos \hat{\phi}_{k}+\hat{e}_{k} \sin \hat{\phi}_{k}, \hat{e}_{k} \perp y_{1},\left\|\hat{e}_{k}\right\|=1, \\
\hat{d}_{k+1} & =y_{1} \cos \hat{\psi}_{k}+\hat{f}_{k} \sin \hat{\psi}_{k}, \hat{f}_{k} \perp y_{1},\left\|\hat{f}_{k}\right\|=1
\end{aligned}
$$

be the orthogonal direct sum decompositions. Then it is known [7] that

$$
\begin{aligned}
\left|\mu_{1}\right| & =O\left(\sin \phi_{k}\right), \\
\sin \hat{\phi}_{k} & \leq c_{1} \sin \phi_{k}
\end{aligned}
$$

with $c_{1}$ a constant.

Similar to Theorem 3, we can derive the following results.

Theorem 8. It holds that

$$
\begin{aligned}
\left|\cos \psi_{k}\right| & =O\left(\sin \phi_{k}\right), \\
\sin \psi_{k} & =1-O\left(\sin ^{2} \phi_{k}\right) .
\end{aligned}
$$


Proof. For the $m$-step Lanczos method for (45), we have $\hat{\xi}_{k+1} \hat{d}_{k+1} \perp \mathcal{K}_{m}\left(B, \mathcal{L}^{-1} u_{k}\right)$. Particularly, it holds that

$$
\hat{d}_{k+1}^{*} \mathcal{L}^{-1} u_{k}=\hat{d}_{k+1}^{*} \mathcal{L}^{-1} u_{k}=0,
$$

from which and $\hat{u}_{k}=\mathcal{L}^{-1} u_{k} /\left\|\mathcal{L}^{-1} u_{k}\right\|$ it follows that $\hat{d}_{k+1}^{*} \hat{u}_{k}=0$. Therefore, from $d_{k+1}=$ $\frac{\mathcal{L} \hat{d}_{k+1}}{\left\|\mathcal{L} \hat{d}_{k+1}\right\|}$ we have

$$
0=\hat{d}_{k+1}^{*} \hat{u}_{k}=\hat{d}_{k+1}^{*} \underbrace{\mathcal{L}^{*} \mathcal{L}^{-*}}_{I} \hat{u}_{k}=\left\|\mathcal{L} \hat{d}_{k+1}\right\|\left\|\mathcal{L}^{-1} u_{k}\right\| d_{k+1}^{*} \mathcal{L}^{-*} \hat{u}_{k}=0
$$

i.e.,

$$
d_{k+1}^{*} \mathcal{L}^{-*} \hat{u}_{k}=0
$$

By definition, we have

$$
\mathcal{L}^{-1}\left(A-\theta_{k} I\right) \mathcal{L}^{-*} y_{1}=\mu_{1} y_{1}
$$

from which it follows that

$$
\left(A-\theta_{k} I\right) \tilde{u}_{k}=\mu_{1} \frac{\mathcal{L} y_{1}}{\left\|\mathcal{L}^{-*} y_{1}\right\|}
$$

with $\tilde{u}_{k}=\mathcal{L}^{-*} y_{1} /\left\|\mathcal{L}^{-*} y_{1}\right\|$. Therefore, by standard perturbation theory and (49), we get

$$
\sin \angle\left(\tilde{u}_{k}, x\right)=O\left(\mu_{1} \frac{\left\|\mathcal{L} y_{1}\right\|}{\left\|\mathcal{L}^{-*} y_{1}\right\|}\right)=O\left(\left|\mu_{1}\right|\right)=O\left(\sin \phi_{k}\right) .
$$

On the other hand, from (50), we have

$$
\sin \hat{\phi}_{k}=\sin \angle\left(\hat{u}_{k}, y_{1}\right)=\sin \angle\left(\mathcal{L}^{-1} u_{k}, y_{1}\right)=O\left(\sin \phi_{k}\right) .
$$

Therefore, we can write

$$
\hat{u}_{k}=y_{1}+O\left(\sin \phi_{k}\right)
$$

which leads to

$$
\mathcal{L}^{-*} \hat{u}_{k}=\mathcal{L}^{-*} y_{1}+O\left(\sin \phi_{k}\right)
$$

Thus, we have

$$
\sin \angle\left(\mathcal{L}^{-*} \hat{u}_{k}, \mathcal{L}^{-*} y_{1}\right)=\sin \angle\left(\mathcal{L}^{-*} \hat{u}_{k}, \tilde{u}_{k}\right)=O\left(\sin \phi_{k}\right)
$$

Since

$$
\angle\left(\mathcal{L}^{-*} \hat{u}_{k}, x\right) \leq \angle\left(\mathcal{L}^{-*} \hat{u}_{k}, \tilde{u}_{k}\right)+\angle\left(\tilde{u}_{k}, x\right),
$$

combining (54) and (55), we get

$$
\sin \angle\left(\mathcal{L}^{-*} \hat{u}_{k}, x\right) \leq \sin \angle\left(\mathcal{L}^{-*} \hat{u}_{k}, \tilde{u}_{k}\right)+\sin \angle\left(\tilde{u}_{k}, x\right)=O\left(\sin \phi_{k}\right) .
$$

Recall that $d_{k+1}=x \cos \psi_{k}+e_{k} \sin \psi_{k}$ and substituting it and the orthogonal direct sum decomposition

$$
\mathcal{L}^{-*} \hat{u}_{k}=\left\|\mathcal{L}^{-*} \hat{u}_{k}\right\|\left(x \cos \angle\left(\mathcal{L}^{-*} \hat{u}_{k}, x\right)+g_{k} \sin \angle\left(\mathcal{L}^{-*} \hat{u}_{k}, x\right)\right)
$$

with $g_{k} \perp x$ into (53). Then following the proof of Theorem 3 , we can get

$$
\begin{aligned}
\left|\cos \psi_{k}\right| & \leq\left|\tan \angle\left(\mathcal{L}^{-*} \hat{u}_{k}, x\right)\right| \\
\sin \psi_{k} & =1-O\left(\sin ^{2} \angle\left(\mathcal{L}^{-*} \hat{u}_{k}, x\right)\right) .
\end{aligned}
$$

Combining them with (56) yields (51) and (52). 
Using this theorem and writing (51) as $\left|\cos \psi_{k}\right| \leq c_{2} \sin \phi_{k}$ with $c_{2}$ a constant, it is direct to extend Theorems 45 in the unpreconditioned Lanczos case to the tuned preconditioned Lanczos case. We have done preliminary numerical experiments and confirmed the theory. Our concerns in this paper are only the convergence theory of the inexact RQI with the unpreconditioned and tuned preconditioned Lanczos, and the pursue of effective tuned preconditioners is beyond the scope of the current paper. We will only report numerical results on the inexact RQI with the unpreconditioned Lanczos.

\section{$5 \quad$ Numerical experiments}

Our numerical experiments were performed on an Intel (R) Core (TM)2 Quad CPU Q9400 $2.66 \mathrm{GHz}$ with main memory $2 \mathrm{~GB}$ using Matlab 7.8.0 with the machine precision $\epsilon=$ $2.22 \times 10^{-16}$ under the Microsoft Windows XP operating system.

We report the numerical results by the inexact RQI with the unpreconditioned Lanczos for computing the smallest eigenpairs of four symmetric (Hermitian) matrices: BCSPWR08 of order 1624, CAN1054 of order 1054, DWT2680 of order 3025 and LSHP3466 of order 3466 [3]. Recall the definition (13) of $\beta$. Note that the bigger the factor $\beta$ is, the worse conditioned $x_{1}$ is. Meanwhile, for $\beta$ big, Theorem 1 and Theorem 4 show that although RQI and the inexact RQI can still converge cubically and quadratically, they may converge more slowly and needs more outer iterations as the factors $2 \beta$ and $\frac{2 \beta \xi}{1-\alpha}$ in (15) and (22) are big. As a reference, we use the Matlab function eig.m to compute $\beta$. We find that DWT2680 and LSHP3466 are considerably more difficult than the other two. We only report the results on the computation of the smallest eigenpair.

Theorems 45 tells us that the cubic asymptotic convergence of the inexact RQI with Lanczos is achieved for $\xi_{k+1}=O\left(\sin \phi_{k}\right)=O\left(\left\|r_{k}\right\|\right)$ when updating $\left(\theta_{k}, u_{k}\right)$ to get $\left(\theta_{k+1}, u_{k+1}\right)$, in the experiments we take

$$
\xi_{k+1} \leq \frac{\left\|r_{k}\right\|}{\|A\|_{1}}
$$

Other stopping criteria have been taken, e.g., $\xi_{k+1} \leq \min \left\{\tau, \tau\left\|r_{k}\right\|\right\}$ with $\tau=0.1$ in [1]. They are essentially the same as (57) and differ only with the scaling factor before $\left\|r_{k}\right\|$. But (57) may be more general as it takes the size of $A$ into account.

We construct the same initial $u_{0}$ for each matrix that is $x_{1}$ plus a reasonably small perturbation generated randomly in a uniform distribution, such that $\left|\lambda_{1}-\theta_{0}\right|<\frac{\left|\lambda_{2}-\lambda_{1}\right|}{2}$. The algorithm stops whenever $\left\|r_{k}\right\|=\left\|\left(A-\theta_{k} I\right) u_{k}\right\| \leq\|A\|_{1}$ tol, where tol $=10^{-14}$ unless stated otherwise. In the experiments, we use the Matlab function symmlq.m to solve the inner linear systems when $\xi_{k}<1$. We should notice that for $\xi_{k} \geq 1$ the Matlab function symmlq.m cannot be applied. Since the Lanczos method behaves irregularly and may nearly break down or break down for indefinite linear systems, that is, $T_{m}$ in (3) is ill conditioned and can be nearly singular and even numerically singular, it may produce bad approximate solutions with large norms and large residual norms $\xi_{k}$ 's for some steps $m$. As far as solving the linear systems is concerned, such approximate solutions have no accuracy and no practical value. In symmlq.m, if such a bad approximate solution emerges, it always outputs the approximate solution as zero and the residual norm $\xi_{k}=1$ simply, telling us nothing! However, we have seen that in the inexact RQI with Lanczos, $\xi_{k} \geq 1$ is allowed. So for our purpose, we have worked out a Lanczos code that uses the Gram-Schmidt with iterative refinement [18] to generate a numerically orthonormal basis of the Krylov subspace $\mathcal{K}_{m}\left(A, u_{k}\right)$ and delivers 'correct' results that the Lanczos method should produce. We point out that our Lanczos code is not optimized but numerically stable.

We report the results obtained by the inexact RQI with Lanczos for choosing $\xi_{k+1}$ as in (57) and fixed $\xi=0.1,1,5$. Based on our theory, the method should asymptotically 
converge quadratically for the $\xi=1,5$ and use almost the same outer iterations as those for $\xi=0.1$. Therefore, the total computational cost may be reduced considerably. For the inexact RQI with Lanczos, the total inner iteration steps "iters", i.e., the total matrixvector products in inner iterations, is a good and reasonable measure of overall performance of the method, as commonly adopted in many cited papers, e.g., [15] and those of Spence and his coworkers.

Tables 144 list the computed results, where iters denotes the number of total inner iteration steps, iter $^{(k)}$ the number of inner iteration steps at the $k$-th outer iteration and the "." denotes the stagnation of symmlq.m at the iter ${ }^{(k)}$-th step. We comment that in symmlq.m the output $i t e r^{(k)}=m-1$, where $m$ is the steps of the Lanczos process.

\begin{tabular}{|c|c|c|c|c|c|c|}
\hline$\xi_{k} \leq \xi$ & $k$ & $\left\|r_{k}\right\|$ & $\sin \phi_{k}$ & $\xi_{k}$ & iter $^{(k)}$ & iters \\
\hline \multirow[t]{3}{*}{$\overline{0(\mathrm{RQI})}$} & 1 & \multirow{3}{*}{$\begin{array}{c}0.0124 \\
3.1 e-8 \\
2.0 e-15\end{array}$} & 0.0036 & & & \\
\hline & 2 & & $8.5 e-8$ & & & \\
\hline & 3 & & $6.6 e-15$ & & & \\
\hline \multirow{3}{*}{$\frac{\left\|r_{k-1}\right\|}{\|A\|_{1}}$} & 1 & \multirow{3}{*}{$\begin{array}{c}0.0071 \\
3.7 e-8 \\
3.0 e-15\end{array}$} & 0.0029 & 0.0367 & 7 & \multirow[t]{3}{*}{1003} \\
\hline & 2 & & $1.2 e-7$ & $4.1 e-4$ & 40 & \\
\hline & 3 & & $3.0 e-15$ & - & 956 & \\
\hline \multirow[t]{3}{*}{0.1} & 1 & \multirow{3}{*}{$\begin{array}{c}0.0090 \\
2.1 e-5 \\
3.3 e-13\end{array}$} & 0.0045 & 0.0950 & 5 & \multirow[t]{3}{*}{300} \\
\hline & 2 & & $3.7 e-5$ & 0.0847 & 24 & \\
\hline & 3 & & $3.2 e-13$ & 0.0754 & 44 & \\
\hline \multirow[t]{4}{*}{1} & 1 & 0.0462 & 0.0165 & 0.7161 & 3 & \multirow[t]{4}{*}{87} \\
\hline & 2 & \multirow{2}{*}{$\begin{array}{l}5.1 e-4 \\
2.3 e-7\end{array}$} & $8.9 e-4$ & 0.8505 & 11 & \\
\hline & 3 & & $2.9 e-7$ & 0.9829 & 26 & \\
\hline & 4 & $1.1 e-14$ & $1.3 e-14$ & - & 47 & \\
\hline \multirow[t]{5}{*}{5} & 1 & 0.1259 & 0.0332 & 2.0694 & 2 & \multirow[t]{5}{*}{87} \\
\hline & 2 & 0.0107 & 0.0064 & 3.0112 & 4 & \\
\hline & 3 & \multirow{3}{*}{$\begin{array}{c}1.9 e-4 \\
1.2 e-7 \\
5.7 e-14\end{array}$} & $2.5 e-4$ & 4.2267 & 13 & \\
\hline & 4 & & $1.6 e-7$ & 4.8117 & 25 & \\
\hline & 5 & & $3.5 e-14$ & 4.8558 & 43 & \\
\hline & & $\begin{array}{ll}m \\
\end{array}$ & " outer iterations & iters & & \\
\hline & & 5 & 110 & 550 & & \\
\hline & & 10 & 21 & 210 & & \\
\hline & & 15 & 10 & 150 & & \\
\hline & & 20 & 7 & 140 & & \\
\hline & & 30 & 5 & 150 & & \\
\hline
\end{tabular}

Table 1: BCSPWR08, $\beta=40.19, \sin \phi_{0}=0.1020$.

Before commenting the experiments, we should remind that in finite precision arithmetic $\left\|r_{k}\right\| /\|A\|_{1}$ can not decline further whenever it reaches a moderate multiple of $\epsilon=$ $2.2 \times 10^{-16}$. Therefore, assuming that the algorithm stops at outer iteration $k$, if $\sin \phi_{k-1}$ or $\left\|r_{k-1}\right\| \leq 10^{-6}$ or $10^{-9}$, then the algorithm may not continue converging cubically or quadratically at the final outer iteration $k$. Another point is that when judging convergence rates, we must take the factor $\beta$ into account. The smaller it is, the more clearly cubic and quadratic convergence exhibits, as indicated by (27) and (29); the bigger it is, the less apparent cubic and quadratic convergence is. So we should precisely base (27) or (29) to judge cubic and quadratic convergence of $\sin \phi_{k}$ or $\left\|r_{k}\right\|$. In this sense, we see from Tables 1 4 that the exact RQI and the inexact RQI with Lanczos for decreasing $\xi_{k}$ converge cubically 


\begin{tabular}{|c|c|c|c|c|c|c|c|}
\hline$\xi_{k} \leq \xi$ & $k$ & \multicolumn{2}{|c|}{$\left\|r_{k}\right\|$} & $\sin \phi_{k}$ & $\xi_{k}$ & iter $^{(k)}$ & iters \\
\hline \multirow{4}{*}{0 (RQI) } & 1 & \multicolumn{2}{|c|}{0.0269} & 0.0110 & & & \\
\hline & 2 & \multicolumn{2}{|c|}{$1.1 e-7$} & $2.1 e-7$ & & & \\
\hline & 3 & \multicolumn{2}{|c|}{$3.2 e-15$} & $5.0 e-15$ & & & \\
\hline & 4 & \multicolumn{2}{|c|}{$2.5 e-15$} & $4.7 e-15$ & & & \\
\hline \multirow{3}{*}{$\frac{\left\|r_{k-1}\right\|}{\|A\|_{1}}$} & 1 & \multicolumn{2}{|c|}{0.0155} & 0.0038 & 0.0403 & 8 & 432 \\
\hline & 2 & \multirow{2}{*}{\multicolumn{2}{|c|}{$\begin{array}{c}1.4 e-7 \\
1.4 e-14\end{array}$}} & $3.1 e-7$ & $3.1 e-4$ & 45 & \\
\hline & 3 & & & $5.0 e-15$ & - & 379 & \\
\hline \multirow[t]{4}{*}{0.1} & 1 & \multicolumn{2}{|c|}{0.0181} & 0.0055 & 0.0796 & 6 & 207 \\
\hline & 2 & \multirow{2}{*}{\multicolumn{2}{|c|}{$\begin{array}{c}3.8 e-6 \\
4.7 e-13\end{array}$}} & $2.2 e-5$ & 0.0679 & 30 & \\
\hline & 3 & & & $2.0 e-13$ & 0.0969 & 51 & \\
\hline & 4 & \multicolumn{2}{|c|}{$6.0 e-15$} & $5.0 e-15$ & - & 120 & \\
\hline \multirow[t]{4}{*}{1} & 1 & \multicolumn{2}{|c|}{0.0624} & 0.0132 & 0.4376 & 4 & 107 \\
\hline & 2 & \multicolumn{2}{|c|}{$5.7 e-4$} & $9.7 e-4$ & 0.9980 & 14 & \\
\hline & 3 & \multicolumn{2}{|c|}{$2.4 e-7$} & $1.3 e-7$ & 0.9561 & 30 & \\
\hline & 4 & \multicolumn{2}{|c|}{$1.7 e-14$} & $8.0 e-14$ & 0.9952 & 53 & \\
\hline \multirow[t]{6}{*}{5} & 1 & \multicolumn{2}{|c|}{0.2238} & 0.0454 & 1.7072 & 2 & 101 \\
\hline & 2 & \multicolumn{2}{|c|}{0.0310} & 0.00946 & 3.7008 & 4 & \\
\hline & 3 & \multicolumn{2}{|c|}{$8.3 e-4$} & 0.0012 & 4.5335 & 11 & \\
\hline & 4 & \multicolumn{2}{|c|}{$1.7 e-6$} & $1.1 e-6$ & 3.6374 & 26 & \\
\hline & 5 & \multicolumn{2}{|c|}{$4.0 e-12$} & $1.5 e-12$ & 3.9095 & 45 & \\
\hline & 6 & \multicolumn{2}{|c|}{$3.4 e-15$} & $2.0 e-14$ & 4.5305 & 13 & \\
\hline & & $m$ & out & er iterations & iters & & \\
\hline & & 5 & & 110 & 550 & & \\
\hline & & 10 & & 21 & 210 & & \\
\hline & & 15 & & 10 & 150 & & \\
\hline & & 20 & & 7 & 140 & & \\
\hline & & 30 & & 5 & 150 & & \\
\hline
\end{tabular}

Table 2: CAN1054, $\beta=88.28, \sin \phi_{0}=0.1008$.

and the method starts to converge quadratically for the given fixed $\xi$ 's after very few outer iterations. Strikingly, for $\xi=1,5$, the method is much more efficient than that for $\xi=0.1$ and for decreasing $\xi_{k}$; the method with $\xi \geq 1$ is four times and twice as fast as that with $\xi=0.1$ for BCSPWR08 and for CAN1054 and DWT2680, respectively. For LSHP3466, the gain is not so great, but the method with $\xi \geq 1$ is still one and a half times as fast as the method with $\xi=0.1$. With the fixed $\xi=1,5$, it is always three to ten times as fast as that with decreasing $\xi_{k}=O\left(\left\|r_{k-1}\right\|\right)$ for the four test matrices. It is seen that for a bigger $\xi$ the method may need a little more outer iterations but it does indeed converge quadratically and is in agreement with quadratic convergence bound (27). Why the method with fixed bigger $\xi_{k}$ 's converges a little more slowly is due to the bigger convergence factor $\frac{2 \beta \xi}{1-\alpha}$ in (22).

Note that the linear systems $\left(A-\theta_{k}\right) w=u_{k}$ 's are Hermitian indefinite and become increasingly worse conditioned and even numerically singular as $\theta_{k} \rightarrow \lambda$ with increasing $k$. So, more inner iteration steps are needed generally for a fixed $\xi$ as $k$ increases. We find that for the difficult DTW2680 and LSHP3466, many more inner iterations are used than those for BCSPWR08 and CAN1054.

We have tested many fixed $\xi$ 's ranging from 10 to 50 for each matrix and found that 


\begin{tabular}{|c|c|c|c|c|c|c|}
\hline$\xi_{k} \leq \xi$ & $k$ & $\left\|r_{k}\right\|$ & $\sin \phi_{k}$ & $\xi_{k}$ & iter $^{(k)}$ & iters \\
\hline \multirow[t]{4}{*}{$0(\mathrm{RQI})$} & 1 & 0.0144 & 0.1188 & & & \\
\hline & 2 & $1.6 e-4$ & 0.0018 & & & \\
\hline & 3 & $5.1 e-10$ & $2.6 e-8$ & & & \\
\hline & 4 & $1.0 e-15$ & $6.7 e-13$ & & & \\
\hline \multirow{4}{*}{$\frac{\left\|r_{k-1}\right\|}{\|A\|_{1}}$} & 1 & 0.0143 & 0.0121 & 0.1171 & 5 & 1512 \\
\hline & 2 & $4.8 e-5$ & $5.1 e-4$ & 0.0019 & 167 & \\
\hline & 3 & $4.2 e-11$ & $9.9 e-10$ & - & 424 & \\
\hline & 4 & $6.1 e-15$ & $6.6 e-13$ & - & 916 & \\
\hline \multirow[t]{4}{*}{0.1} & 1 & 0.0123 & 0.0104 & 0.0830 & 6 & 955 \\
\hline & 2 & $5.1 e-5$ & $1.1 e-4$ & 0.0972 & 93 & \\
\hline & 3 & $1.3 e-11$ & $1.5 e-10$ & 0.0986 & 267 & \\
\hline & 4 & $5.0 e-15$ & $6.6 e-13$ & $0.1109\left(^{*}\right)$ & 589 & \\
\hline \multirow[t]{4}{*}{1} & 1 & 0.0419 & 0.0265 & 0.4842 & 3 & 402 \\
\hline & 2 & $6.7 e-4$ & 0.0041 & 0.9424 & 15 & \\
\hline & 3 & $8.6 e-7$ & $1.2 e-5$ & 0.9667 & 117 & \\
\hline & 4 & $1.9 e-12$ & $2.2 e-11$ & 0.9276 & 267 & \\
\hline \multirow[t]{6}{*}{5} & 1 & 0.0851 & 0.0387 & 1.0223 & 2 & 535 \\
\hline & 2 & 0.0092 & 0.0125 & 3.8250 & 5 & \\
\hline & 3 & $2.8 e-4$ & 0.0027 & 4.8280 & 19 & \\
\hline & 4 & $1.1 e-6$ & $1.3 e-5$ & 4.7719 & 108 & \\
\hline & 5 & $1.3 e-11$ & $2.6 e-10$ & 4.8329 & 238 & \\
\hline & 6 & $7.0 e-15$ & $5.8 e-13$ & 4.9521 & 163 & \\
\hline & & $m$ & uter iterations & iters & & \\
\hline & & 10 & 268 & 2680 & & \\
\hline & & 20 & 55 & 1100 & & \\
\hline & & 30 & 30 & 900 & & \\
\hline & & 40 & 17 & 680 & & \\
\hline & & 50 & 11 & 550 & & \\
\hline & & 60 & 11 & 660 & & \\
\hline
\end{tabular}

Table 3: DWT2680, tol $=10^{-12}, \beta=2295.6, \sin \phi_{0}=0.1095$.

the method converges quadratically. $\xi \geq 10$ does not satisfy condition (21) for quadratic convergence but is a moderate multiple of $\frac{1}{\sin \phi_{0}}$. The algorithm with these $\xi$ behaves almost the same as that with $\xi=1,5$ and uses a little more outer iterations and comparable total inner iteration steps iters.

Unlike quadratic convergence where it is not necessary to estimate $\xi$ accurately, for linear convergence, we see that conditions (25) and (33) heavily depend on and are sensitive to the a-priori $\beta$. So it appears impossible to design a practical criterion robustly and reliably unless a good estimate on $\beta$ is available in advance. Note that the convergence of the method allows $\xi_{k+1}$ to increase up to $O\left(\frac{1}{\left\|r_{k}\right\|}\right)$ as outer iterations proceed. Therefore, we may implement the inexact RQI with Lanczos for certain fixed inner iteration steps $m$ 's. Doing so is based on a not very stringent expectation that resulting $\xi_{k+1}$ 's are not too large and at least obey one of (25) and (33). Of course, if $T_{m}$ is too ill conditioned, it is possible for $\xi_{k+1}$ to be too large and exceed bounds (25) and (33). We have tested several $m$ 's for each test matrix, see Tables 14 for results. Figure 1 displays convergence processes of the inexact RQI with Lanczos for various fixed $m$ 's. 


\begin{tabular}{|c|c|c|c|c|c|c|c|}
\hline$\xi_{k} \leq \xi$ & $k$ & \multicolumn{2}{|c|}{$\left\|r_{k}\right\|$} & $\sin \phi_{k}$ & $\xi_{k}$ & iter $^{(k)}$ & iters \\
\hline \multirow[t]{4}{*}{0 (RQI) } & $\overline{1}$ & \multicolumn{2}{|c|}{0.0149} & 0.1716 & & & \\
\hline & 2 & \multirow{2}{*}{\multicolumn{2}{|c|}{$\begin{array}{l}4.0 e-4 \\
1.2 e-8\end{array}$}} & 0.0056 & & & \\
\hline & 3 & & & $8.9 e-7$ & & & \\
\hline & 4 & \multicolumn{2}{|c|}{$2.0 e-15$} & $4.0 e-13$ & & & \\
\hline \multirow{4}{*}{$\frac{\left\|r_{k-1}\right\|}{\|A\|_{1}}$} & 1 & \multicolumn{2}{|c|}{0.0102} & 0.0097 & 0.0874 & 6 & 1717 \\
\hline & 2 & \multicolumn{2}{|c|}{$3.9 e-5$} & $1.2 e-4$ & 0.0014 & 201 & \\
\hline & 3 & \multicolumn{2}{|c|}{$1.2 e-9$} & $4.5 e-8$ & - & 497 & \\
\hline & 4 & \multicolumn{2}{|c|}{$5.2 e-15$} & $6.1 e-13$ & - & 1013 & \\
\hline \multirow[t]{4}{*}{0.1} & 1 & \multicolumn{2}{|c|}{0.0102} & 0.0098 & 0.0874 & 6 & 651 \\
\hline & 2 & \multicolumn{2}{|c|}{$4.2 e-4$} & $5.4 e-4$ & 0.0990 & 102 & \\
\hline & 3 & \multicolumn{2}{|c|}{$4.5 e-8$} & $2.8 e-7$ & 0.0948 & 256 & \\
\hline & 4 & \multicolumn{2}{|c|}{$5.3 e-13$} & $5.6 e-11$ & 0.0965 & 287 & \\
\hline \multirow[t]{4}{*}{1} & 1 & \multicolumn{2}{|c|}{0.0408} & 0.0251 & 0.5335 & 3 & 424 \\
\hline & 2 & \multicolumn{2}{|c|}{$6.4 e-4$} & 0.0036 & 0.9685 & 15 & \\
\hline & 3 & \multicolumn{2}{|c|}{$7.6 e-7$} & $1.9 e-5$ & 0.9770 & 123 & \\
\hline & 4 & \multicolumn{2}{|c|}{$2.7 e-12$} & $2.9 e-11$ & 0.9907 & 283 & \\
\hline \multirow[t]{6}{*}{5} & 1 & \multicolumn{2}{|c|}{0.0779} & 0.0370 & 1.0528 & 2 & 444 \\
\hline & 2 & \multicolumn{2}{|c|}{0.0088} & 0.0128 & 4.2424 & 5 & \\
\hline & 3 & \multicolumn{2}{|c|}{$2.4 e-4$} & 0.0021 & 4.4646 & 21 & \\
\hline & 4 & \multicolumn{2}{|c|}{$7.0 e-7$} & $2.8 e-5$ & 4.6415 & 121 & \\
\hline & 5 & \multicolumn{2}{|c|}{$1.9 e-11$} & $2.4 e-10$ & 4.6506 & 146 & \\
\hline & 6 & \multicolumn{2}{|c|}{$1.0 e-14$} & $3.8 e-13$ & 4.6805 & 149 & \\
\hline & & $m$ & out & r iterations & iters & & \\
\hline & & 10 & & 278 & 2780 & & \\
\hline & & 20 & & 54 & 1080 & & \\
\hline & & 30 & & 32 & 960 & & \\
\hline & & 40 & & 19 & 760 & & \\
\hline & & 50 & & 13 & 650 & & \\
\hline & & 60 & & 11 & 660 & & \\
\hline
\end{tabular}

Table 4: LSHP3466, tol $=10^{-12}, \beta=2613.1, \sin \phi_{0}=0.1011$.

We find that the method works very well and robustly. In terms of iters, it is seen from the tables that the overall efficiency of the inexact RQI with Lanczos for fixed small $m$ 's is comparable to that of the method with given fixed $\xi$ 's, except $m=5$ for the easy problems BCSPWR08 and CAN1054 and $m=10$ for the relatively difficult problems DWT2680 and LSHP3466. Furthermore, we observe that for BCSPWR08 and CAN1054 the inexact RQI with Lanczos converges almost as fast as the exact RQI for $m=30$, and for the difficult problems DWT2680 and LSHP3466 we need to properly increase $m$ to achieve fast outer convergence. As expected, it is not surprising from the figures that a five-step and at most ten-step Lanczos method for the inner linear systems is enough to ensure the convergence of the inexact RQI with Lanczos. However, for the difficult BWT2680 and LSHP3466, the inexact RQI with Lanczos fails to converge when $m=5$. The reason is that some $\xi_{k+1}$ 's are too big and violate the linear convergence conditions. Finally, we should point out that although the Lanczos method with a smaller $m$ usually produces a bigger $\xi_{k+1}$ and makes the inexact RQI use more outer iterations, the total inner iteration steps iters may not increase. 

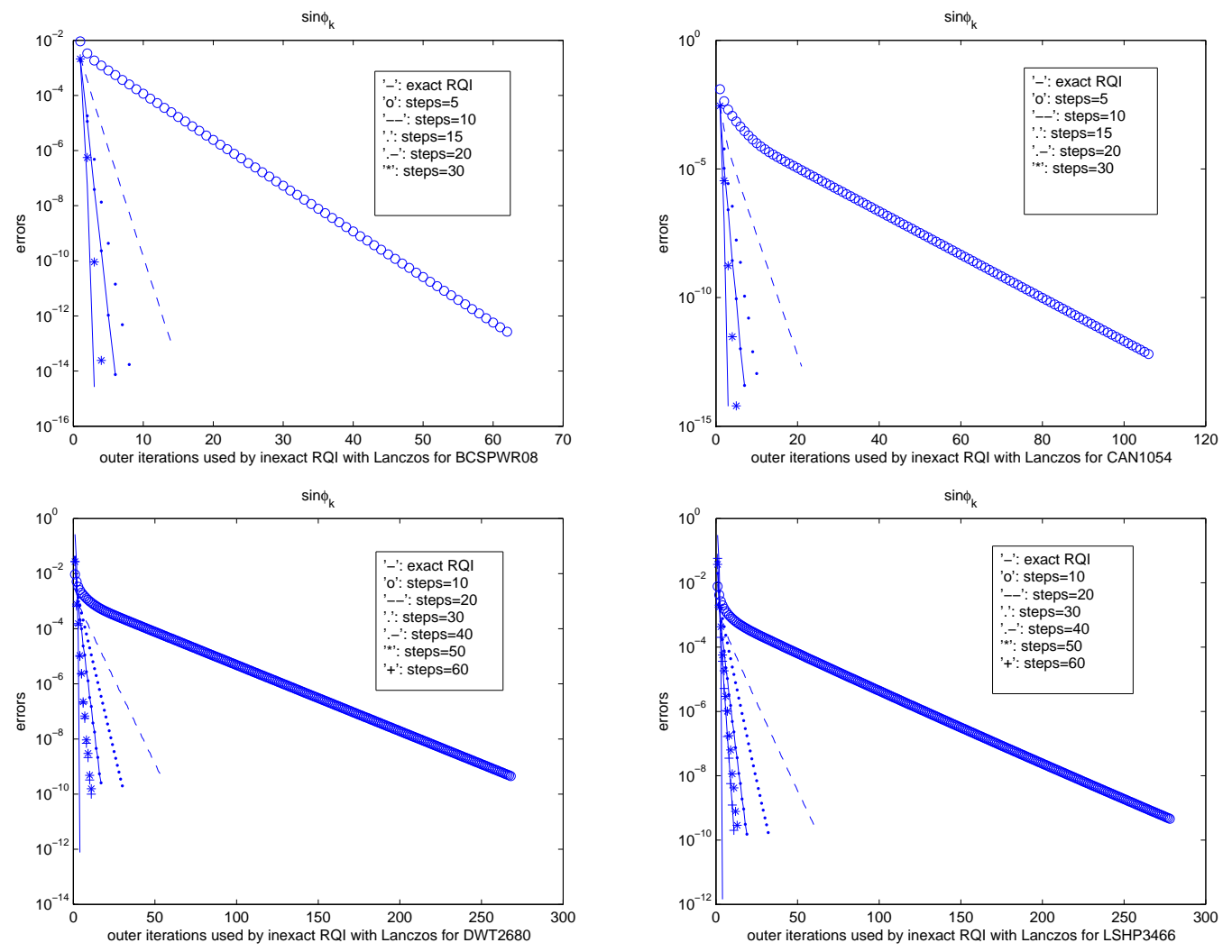

Figure 1: The inexact RQI with Lanczos for varying inner iteration steps

To see how big $\xi_{k+1}$ 's may be as outer iterations converge, we display the curves of $\xi_{k+1}$ 's versus $\frac{1}{\sin \phi_{k}}$ 's for some fixed $m$ 's in Figure 2, We remark that $\xi_{k+1}$ in the figure should correspond to $\xi_{k}$ in our context.

It is clear from Figure 2 that the inexact RQI with Lanczos works very well and $\sin \phi_{k}$ tends to zero smoothly and quickly, that is, $\frac{1}{\sin \phi_{k}}$ tends to infinity smoothly and quickly, though most of $\xi_{k+1}$ 's are much bigger than one and some of them are near $\frac{1}{\sin \phi_{k}}$ and can be as big as $10^{4} \sim 10^{7}$ ! Furthermore, we observe that almost all $\xi_{k+1}$ 's are smaller than $\frac{1}{\sin \phi_{k}}$ and the only exception is $\xi_{6}>\frac{1}{\sin \phi_{6}}$ for BWT2680.

We also computed some other eigenpairs of each test matrix. We observed similar behavior and confirmed our theory. However, when an interior eigenpair was required, we often needed much more iters. This is because the shifted inner linear systems can be highly indefinite (i.e., each shifted matrix has many positive and negative eigenvalues) and may be hard to solve. If the smallest or largest eigenpair is required, then $A-\theta_{k} I$ has only one negative or positive eigenvalue, assuming that $A$ only has simple eigenvalues. As a consequence, after the smallest Ritz value converges to the smallest eigenvalue $\lambda-\theta_{k}$ of $A-\theta_{k} I$, the Lanczos method will behave as if $A-\theta_{k} I$ is positive or negative definite, so that it converges smoothly after the smallest Ritz value converges. Comparing with the computation of the smallest or largest eigenpair, we found that we must take a considerably bigger fixed inner iteration steps $m$ to make the method converge correctly when an interior eigenpair was desired. For a descriptive analysis, see, e.g., [4, 17, 11, 21]. 

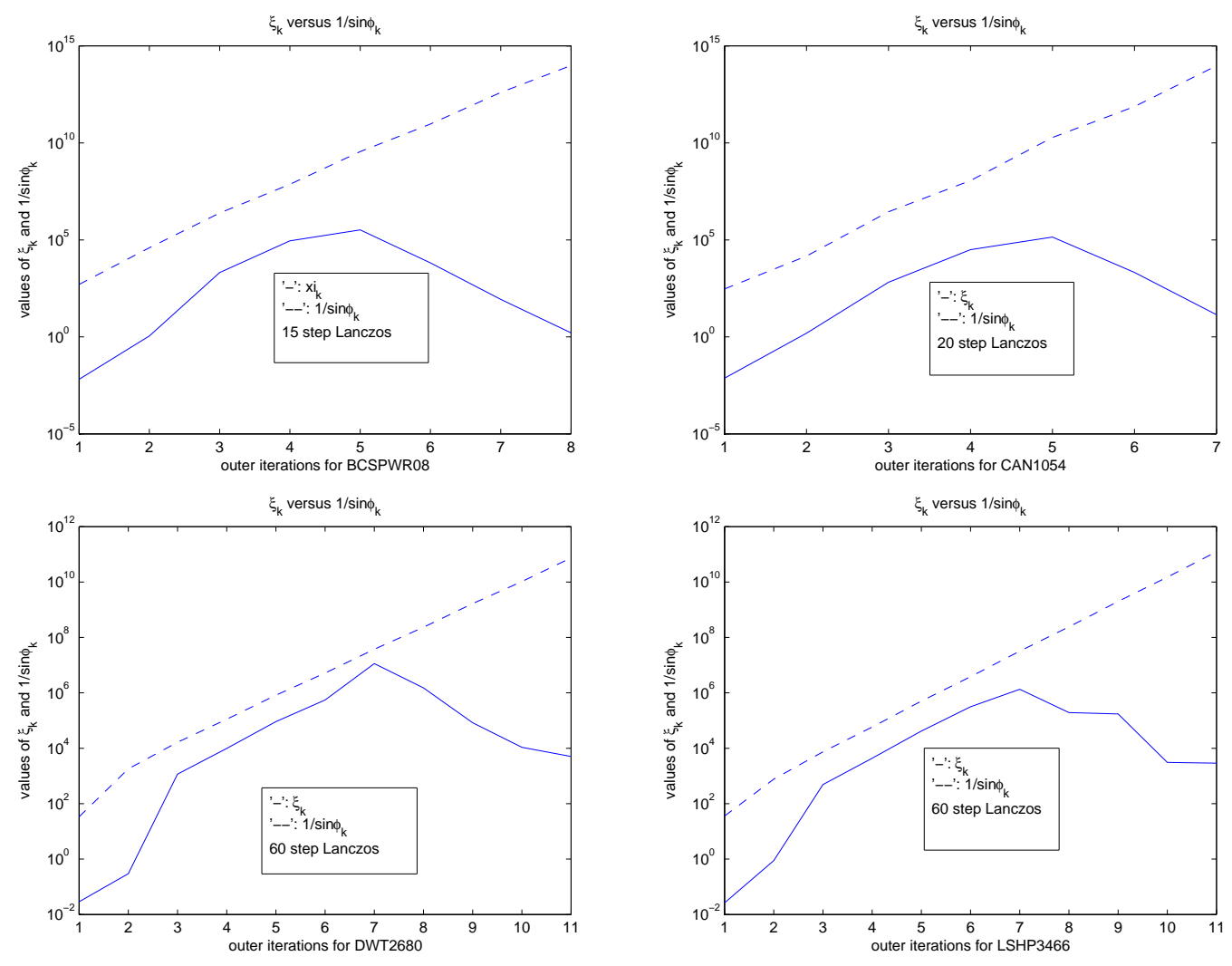

Figure 2: $\xi_{k+1}$ versus $\frac{1}{\sin \phi_{k}}$.

\section{Conclusions}

We have considered the convergence of the inexact RQI with the unpreconditioned and tuned preconditioned Lanczos methods and have established a number of results. These results show how inner tolerance affects accuracy of outer iterations and provide practical criteria on how to best control inner tolerance to achieve the quadratic asymptotic convergence of the inexact RQI. It is the first time to appear surprisingly that the inexact RQI with Lanczos converge quadratically provided $\xi_{k+1} \leq \xi$ with $\xi$ a constant being allowed bigger than one. This is both attractive and exciting as we can implement the method much more effectively than ever before. Numerical experiments have confirmed our theory.

Perspectively, since the inexact RQI has intimate relations with the simplified JacobiDavidson method and the former is mathematically equivalent to the latter when a GalerkinKrylov type solver, e.g., the Lanczos method, is used for solving the linear systems, we can use the convergence theory developed here for the inexact RQI to help understand the inexact simplified JD method. In this respect, Simoncini and Eldén [15] have proved the mathematical equivalence of the two methods, and Freitag and Spence [6] have given a further analysis on the methods with preconditioned Lanczos solves. Based on the equivalence, it is significant to extend our theory in this paper to the simplified Jacobi-Davidson method with unpreconditioned and preconditioned Lanczos inner solves. A similar relaxation on $\xi_{k+1}$ is expected. Meanwhile, the inexact inverse iteration is a simpler variation of the inexact RQI, where varying $\theta_{k}$ 's are fixed to be a constant $\sigma$, leading to different convergence behavior. Thus, a specific analysis is needed. It is likely to exploit the analysis approach used in this paper to study the inexact inverse iteration. Finally, although we 
have restricted to the Hermitian case, the analysis approach in this paper may be applied to the inexact RQI for the non-Hermitian eigenvalue problem, where the Arnoldi method is used for non-Hermitian inner linear systems.

\section{References}

[1] J. Berns-Müller, I. G. Graham and A. Spence, Inexact inverse iteration for symmetric matrices, Linear Algebra Appl., 416 (2006), pp. 389-413.

[2] J. Berns-Müller and A. Spence, Inexact inverse iteration with variable shift for nonsymmetric generalized eigenvalue problems, SIAM J. Matrix Anal. Appl., 28 (2006), pp. 1069-1082.

[3] I. S. Duff, R. G. Grimes and J. G. Lewis, User's Guide for the Harwell-Boebing sparse matrix collection (Release 1), Tech. Rep., RAL-92-086, Rutherford Appleton Laboratory, UK, 1992. Data available at http://math.nist.gov/MarketMatrix.

[4] M. A. Freitag and A. Spence, Convergence of inexact inverse iteration with application to preconditioned iterative solves. BIT, (47) (2007), pp. 27-44.

[5] M. A. Freitag and A. Spence, Convergence theory for inexact inverse iteration applied to the generalised nonsymmetric eigenproblem, Electron. Trans. Numer. Anal., (28) (2007), pp. 40-64.

[6] M. A. Freitag and A. Spence, Rayleigh quotient iteration and simplified JacobiDavidson method with preconditioned iterative solves, Linear Algebra Appl., 428 (2008), pp. 2049-2060.

[7] M. A. Freitag and A. Spence, A tuned preconditioner for inexact inverse iteration applied to Hermitian eigenvalue problems, IMA J. Numer. Anal., 28 (2008), pp. 522551.

[8] G. H. Golub and C. F. van Loan, Matrix Computations, The John Hopkins University Press, Baltimore, London, 1996.

[9] Z. Jia, On convergence of the inexact Rayleigh quotient iteration with MINRES, arXiv: math/0906.2238v3, 2010.

[10] Y. Notay, Convergence analysis of inexact Rayleigh quotient iteration, SIAM J. Matrix Anal. Appl., 24 (2003), pp. 627-644.

[11] C. C. Paige, B. N. Parlett and H. A. van der Vorst, Approximate solutions and eigenvalue bounds from Krylov subspaces, Numer. Linear Algebra Appl., 2 (1995), pp. 115134.

[12] C. C. Paige and M. A. Saunders, Solution of sparse indefinite systems of linear equations, SIAM J. Numer. Anal., 12 (1975), pp. 617-629.

[13] B. N. Parlett, The Symmetric Eigenvalue Problem, SIAM, Philadelphia, PA, 1998.

[14] Y. Saad, Iterative Methods of Large Sparse Linear Systems, 2nd Edition, SIAM, Philadelphia, PA, 2003.

[15] V. Simoncini and L. Eldén, Inexact Rayleigh quotient-type methods for eigenvalue computations, BIT, 42 (2002), pp. 159-182. 
[16] P. Smit and M. H. C. Paardekooper, The effects of inexact solvers in algorithms for symmetric eigenvalue problems, Linear Algebra Appl., 287 (1999), pp. 337-357.

[17] A. Stathopoulos and Y. Saad, Restarting techniques for the (Jacobi-)Davidson eigenvalue methods, Electr. Trans. Numer. Anal., 7 (1998), pp. 163-181.

[18] G. W. Stewart, Matrix Algorithms Vol. II: Eigensystems, SIAM, Philadelphia, PA, 2001.

[19] J. van den Eshof, The convergence of Jacobi-Davidson iterations for Hermitian eigenproblems, Numer. Linear Algebra Appl., 9 (2002), pp. 163-179.

[20] H. A. van der Vorst, Computational Methods for Large Eigenvalue Problems, In P. G. Ciarlet and J. L. Lions (eds), Handbook of Numerical Analysis, Vol. VIII, NorthHolland, Elsevier, pp. 3-179, 2002.

[21] F. Xue and H. Elman, Convergence analysis of iterative solvers in inexact Rayleigh quotient iteration, SIAM. J. Matrix Anal. Appl., 31 (2009), pp. 877-899.

[22] F. Xue and D. B. Szyld, Efficient preconditioned inner solves for inexact Rayleigh quotient iteration and their connections to the single-vector Jacobi-Davidson method, SIAM J. Matrix Anal. Appl,, accepted. 